\title{
Chemistry and Biological Activity of Alkaloids from the Genus Lycoris (Amaryllidaceae)
}

\section{Lucie Cahlíková *(D), Kateřina Breiterová(i) and Lubomír Opletal}

ADINACO Research Group, Department of Pharmaceutical Botany, Faculty of Pharmacy, Charles University, Heyrovského 1203, 50005 Hradec Králové, Czech Republic; breiterk@faf.cuni.cz (K.B.); opletal@faf.cuni.cz (L.O.)

* Correspondence: cahlikova@faf.cuni.cz; Tel.: +420-495067311

Academic Editor: John C. D'Auria

Received: 20 September 2020; Accepted: 17 October 2020; Published: 19 October 2020

\begin{abstract}
Lycoris Herbert, family Amaryllidaceae, is a small genus of about 20 species that are native to the warm temperate woodlands of eastern Asia, as in China, Korea, Japan, Taiwan, and the Himalayas. For many years, species of Lycoris have been subjected to extensive phytochemical and pharmacological investigations, resulting in either the isolation or identification of more than 110 Amaryllidaceae alkaloids belonging to different structural types. Amaryllidaceae alkaloids are frequently studied for their interesting biological properties, including antiviral, antibacterial, antitumor, antifungal, antimalarial, analgesic, cytotoxic, and cholinesterase inhibition activities. The present review aims to summarize comprehensively the research that has been reported on the phytochemistry and pharmacology of the genus Lycoris.
\end{abstract}

Keywords: Amaryllidaceae; Lycoris; Lycoris radiata; folk medicine; alkaloids; biological activity

\section{Introduction}

Plants of the Amaryllidaceae family, which consists of about 85 genera and 1100 species, are distributed over the tropical and warm regions of the world. They have been extensively used as folk medicines to treat various diseases in many countries and areas [1-3]. Chemically, the Amaryllidaceae family is known for its unique alkaloid constituents, named Amaryllidaceae alkaloids (AAs), which display a wide range of biological activities including acetylcholinesterase (AChE) and butyrylcholinesterase (BuChE) inhibition effects, and antitumor, antifungal, antibacterial, antiviral, and antimalarial properties [1,4-6]. The most known representative of AAS is galanthamine, which is currently used for the treatment of early and intermediate states of Alzheimer's disease (AD) [7].

The present review summarizes phytochemical studies carried out on the genus Lycoris, focusing on the occurrence, isolation, identification and biological activities of its alkaloids. Lycoris species have been used for a long time in traditional medicine.

\section{Genus Lycoris: Occurrence, Ethnobotany}

The genus Lycoris Herbert consists of about 20 species which are distributed in the moist warm temperate woodlands of eastern Asia, as in China, Korea, Japan, Taiwan, and the Himalaya $[8,9]$. The genus was established by Herbert in 1821 [10], and L. aurea has been assigned as the type species. In the mid-19th to early 20th centuries, nine new species were published by various European authors $(L$. africana, L. straminea, L. sewerzowii, L. squamigera, L. sanguinea, L. terraccianii, L. sprengeri, L. incarnata and L. argentea). In the first half of the 20th century three new species from Japan were described (L. albiflora, L. koreana and L. kiusiana) [10]. After that, American botanist Hamilton P. Traub recorded 10 new taxa (two with Moldenke) of Lycoris, based mainly on materials introduced from China, Japan and cultivated 
in American gardens (L. rosea, L. haywardii, L. caldwelii, L. houdyshelii and L. $\times$ woodii, L. chinensis, L. elsiae, L. $\times$ lajolla, L. $\times$ jacksoniana, L. josephinae) [10]. American horticulturist Hayward published, in 1957, details of a new species, L. traubii. About 15 years later, Chinese botanists recorded four new species of Lycoris from China: L. longituba, L. anhuiensis, L. guangxiensis, and L. shaanxiensis [10]. In the 1990s, new species from Korea were described by Korean botanists (L. flavescens, and L. chejuensis) [8,10]. However, some of these species have been reduced to synonyms, some to intraspecific rank, and still others are cultivars or hybrids, since Lycoris species easily hybridize with each other [10]. Plants of the genus Lycoris are extensively cultivated as ornamental plants, especially in China and Japan, and also in other warm temperate regions of the world. Over 230 cultivars have been selected for garden use. Currently, The Plant List accepts twenty-three Lycoris species [11].

Plants of the genus Lycoris have been used for a long time in traditional medicine. The bulbs of L. radiata were used as a traditional Chinese herbal medicine to treat sore throats, carbuncle, cancer, suppurative wounds, poliomyelitis, mastitis, tympanitis, ulcers, and neurodegenerative diseases like AD, but generally, L. radiata has been considered to be toxic [12-16]. In the Compendium of Materia Medica in China, it is recorded that L. radiata can also be used as an antidote to poison, relieve inflammation, alleviate pain, and act as a diuretic drug [17]. L. sprengeri is mainly distributed in the lower reaches of the Yangtze River, and its bulbs have also been used in Chinese medicine for the treatment of similar diseases as L. radiata [18]. The crushed bulbs of L. aurea, which is endemic to the southwest district of Hubei Province in China, have been used as a poultice for treating burns, scalds and ulcers [19].

\section{Phytochemistry of the Genus Lycoris}

Of the accepted 23 Lycoris species, fourteen have been chemically investigated (Table 1). Major attention within the reported phytochemical studies has been given to the study of alkaloids, since they are the most studied constituents of this genus, and only little attention has been given to other components. The AAs are largely restricted to the family Amaryllidaceae, specifically the subfamily Amaryllidoideae [20]. They are derived from the aromatic acids phenyalanine and tyrosine, which are used to produce key intermediates in the biosynthesis of the AA 4'-O-methylnorbelladine [21,22]. According to the name of this key intermediate, this biosynthetic pathway of AAs is called the norbelladine pathway [23]. Recently, several review articles provide detailed coverage of the biosynthesis of AAs [1,23-26], and thus we will not repeat this in the current review. Altogether, 116 AAs of various structural types have been isolated in either pure form or identified by different analytical methods (e.g., GC-MS or HPLC-MS) in the studied Lycoris plants (Table 1; Figures 1-5). The reported alkaloids belong to the belladine (1), crinine (2-6), galanthamine (7-21), galanthindole (22), haemanthamine (23-34), homolycorine (35-59), hostasinine (60), ismine (61), lycorine (62-92), montanine (93-98), narciclasine (99-107) and tazettine (108-116) structural types (Table 1, Figures 1-5). Moreover, 12 alkaloids (117-128) of other structural cores have been reported in some Lycoris species (Table 1). 
<smiles>COC1=CC=C2C=C(O1)N(CC[C@H](O)c1ccc(O)cc1)C2</smiles>

(1)

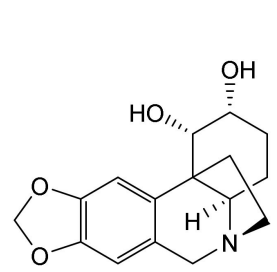

(2)<smiles>COc1ccc2c3c1OC1=C[C@H](O)C=CC13CCN(C)C2</smiles>

Galanthamine-type

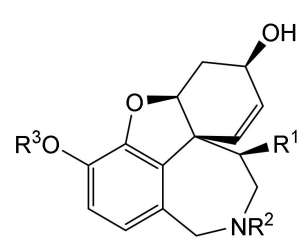

(8) $\mathrm{R}^{1}=\mathrm{OH}, \mathrm{R}^{2}=\mathrm{R}^{3}=\mathrm{Me}$ (9) $R^{1}=R^{2}=H, R^{3}=M e$ (10) $R^{1}=H, R^{2}=A$ llyl, $R^{3}=M e$ (13) $R^{1}=H, R^{2}=R^{3}=M e$ (21) $R^{1}=H, R^{2}=M e, R^{3}=H$<smiles>[R6]c1ccc2c3c1OCC3=CC[N+](C)([O-])C2</smiles>
(17) $\mathrm{R}=\mathrm{Me}$<smiles>[CH+]1CCCCC1</smiles>

(20)<smiles>Cc1ccc2c3c1CC1CC(=O)CC3O[C@H]1CCN(C)C2</smiles>

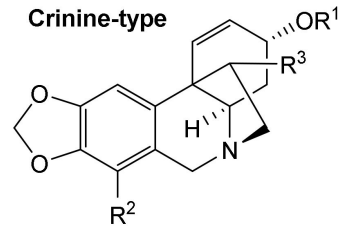

(3) $\mathrm{R}^{1}=\mathrm{Me}, \mathrm{R}^{2}=\mathrm{OMe}, \mathrm{R}^{3}=\mathrm{OH}$ (4) $R^{1}=R^{2}=R^{3}=H$<smiles>COc1ccc2c3c1O[C@H]1C[C@H](O)CC[C@@]31CC[N+](C)(C)C2</smiles>

(11) $\mathrm{O}^{-}$

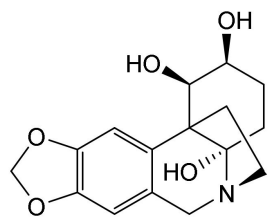

(5)

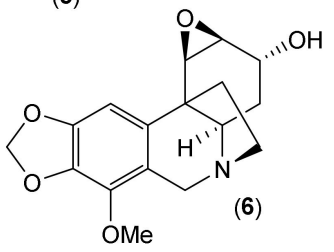

Figure 1. Amaryllidaceae alkaloids of belladine-, crinine-, and galanthamine-type reported in Lycoris species.

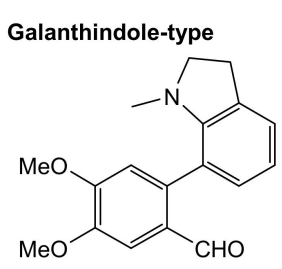

(22)

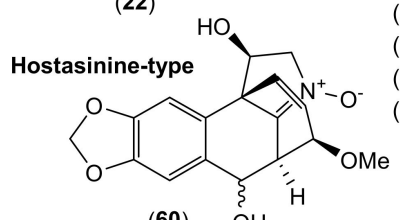

(60) $\mathrm{OH}$

Ismine-type<smiles>CCc1cc2c(cc1-c1ccccc1NC)OCO2</smiles>

(61) $\mathrm{OH}$

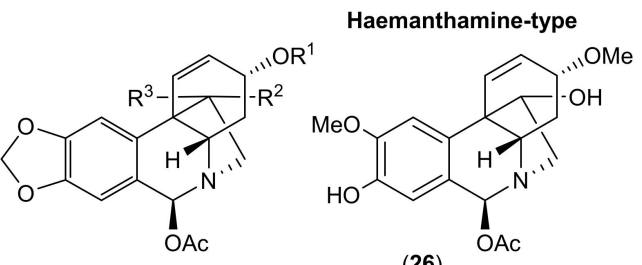

(26)

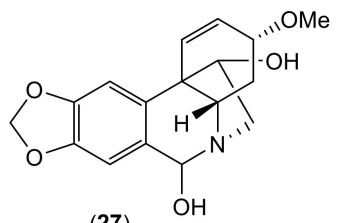

(27)

$\mathrm{R}^{1}=\mathrm{H}, \mathrm{R}^{2}=\mathrm{H}, \mathrm{R}^{3}=\mathrm{OH}$

(24) $\mathrm{R}^{1}=\mathrm{Me}, \mathrm{R}^{2}=\mathrm{H}, \mathrm{R}^{3}=\mathrm{OH}$

(25) $R^{1}=\mathrm{Ac}, \mathrm{R}^{2}=\mathrm{H}, \mathrm{R}^{3}=\mathrm{OH}$

(28) $R^{1}=\mathrm{Me}, \mathrm{R}^{2}=\mathrm{OH}, \mathrm{R}^{3}=\mathrm{H}$

(18) $R^{1}=\mathrm{Me}, \mathrm{R}^{2}=\mathrm{Me}$

(19) $R^{1}=H, R^{2}=M e$

(16)

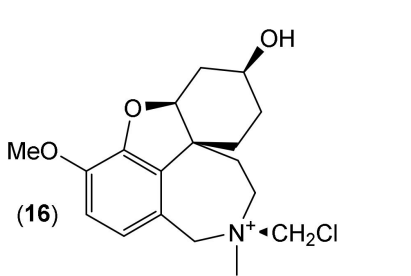

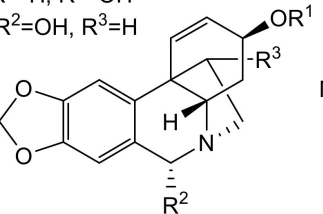

(29) $\mathrm{R}^{1}=\mathrm{Me}, \mathrm{R}^{2}=\mathrm{H}, \mathrm{R}^{3}=\mathrm{OH}$

(30) $\mathrm{R}^{1}=\mathrm{Me}, \mathrm{R}^{2}=\mathrm{OH}, \mathrm{R}^{3}=\mathrm{OH}$

(33) $\mathrm{R}^{1}=\mathrm{R}^{2}=\mathrm{H}, \mathrm{R}^{3}=\mathrm{OH}$

(34) $R^{1}=R^{2}=R^{3}=H$

Figure 2. Amaryllidaceae alkaloids of galanthindole-, haemanthamine-, hostasinine-, and ismine-type reported in Lycoris species. 


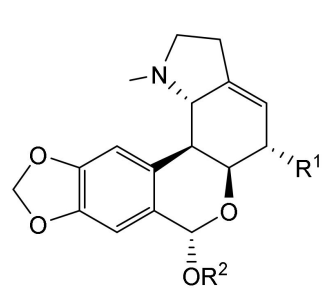

(35) $\mathrm{R}^{1}=\mathrm{OMe}, \mathrm{R}^{2}=\mathrm{Et}$ (36) $\mathrm{R}^{1}=\mathrm{OMe}, \mathrm{R}^{2}=\mathrm{Me}$ (37) $\mathrm{R}^{1}=\mathrm{OH}, \mathrm{R}^{2}=\mathrm{Me}$ (38) $\mathrm{R}^{1}=\mathrm{OH}, \mathrm{R}^{2}=\mathrm{Bu}$ (39) $\mathrm{R}^{1}=\mathrm{OH}, \mathrm{R}^{2}=\mathrm{H}$ (40) $\mathrm{R}^{1}=\mathrm{R}^{2}=\mathrm{R}^{3}=\mathrm{H}$ (56) $\mathrm{R}^{1}=\mathrm{OH}, \mathrm{R}^{2}=\mathrm{Et}$ (59) $\mathrm{R}^{1}=\mathrm{OMe}, \mathrm{R}^{2}=\mathrm{H}$<smiles>COc1cc2c(cc1OC)[C@H]1[C@@H]3C(=CC[C@H]3O[C@H]2OC)CC[N+]1(C)[O-]</smiles>

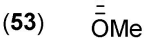<smiles>[R]c1cc2c3c(c1[R])-c1cc4c(cc1C([R])N4CC3)OCO2</smiles>

(70) $R^{1}=R^{2}=R^{3}=H$ (82) $\mathrm{R}^{1}=\mathrm{H}, \mathrm{R}^{2}=\mathrm{OH}, \mathrm{R}^{3}=\mathrm{O}$ (83) $R^{1}=R^{2}=O H, R^{3}=O$

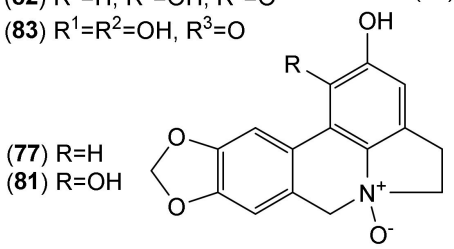

Homolycorine-type<smiles>[R20]c1cc2c(cc1[R20])[C@@H]1[C@@H]3C(=C[C@H]([R1])[C@@H]3OC2=O)CC[N+]1(C)O</smiles><smiles>[R20]Oc1cc2c(cc1O[R20])[C@H]1[C@@H](OC2=O)[C@@H]([R7])C=C[C@H]2CCN(C)[C@H]21</smiles>

(41) $\mathrm{R}^{1}=\mathrm{OH}, \mathrm{R}^{2}=\mathrm{H}, \mathrm{R}^{3}=\mathrm{Me}$

(42) $R^{1}=R^{2}=\mathrm{H}, \mathrm{R}^{3}=\mathrm{Me}$

(46) $\mathrm{R}^{1}=\mathrm{H}, \mathrm{R}^{2}+\mathrm{R}^{3}=\mathrm{CH}_{2}$

(47) $\mathrm{R}^{1}=\mathrm{H}, \mathrm{R}^{2}=\mathrm{Ac}, \mathrm{R}^{3}=\mathrm{Me}$

(48) $R^{1}=\mathrm{H}, \mathrm{R}^{2}=\mathrm{R}^{3}=\mathrm{Me}$

(58) $\mathrm{R}^{1}=\mathrm{OH}, \mathrm{R}^{2}+\mathrm{R}^{3}=\mathrm{CH}_{2}$
(43) $R^{1}=R^{2}=H, R^{3}=M e$
(44) $R^{1}=H, R^{2}=\mathrm{Me}, \mathrm{R}^{3}=\mathrm{H}$
(45) $\mathrm{R}^{1}=\mathrm{OH}, \mathrm{R}^{2}=\mathrm{Me}, \mathrm{R}^{3}=\mathrm{H}$
(49) $\mathrm{R}^{1}=\mathrm{H}, \mathrm{R}^{2}=\mathrm{R}^{3}=\mathrm{Me}$
(57) $\mathrm{R}^{1}=\mathrm{OH}, \mathrm{R}^{2}+\mathrm{R}^{3}=\mathrm{CH}_{2}$<smiles>[R]C1C=C2CCN(C)[C@@H]3c4cc(OC)c(OC)cc4[C@@H](OC1[R2])[C@H]23</smiles>

(50) $R^{1}=H, R^{2}=B u$

(51) $R^{1}=H, R^{2}=E t$

(52) $\mathrm{R}^{1}=\mathrm{H}, \mathrm{R}^{2}=\mathrm{Me}$

(54) $R^{1}=\mathrm{OMe}, \mathrm{R}^{2}=\mathrm{Me}$

(55) $R^{1}=R^{2}=H$<smiles>O[C@H]1CC2CCN3Cc4cc5c(cc4[C@H]([C@H]23)[C@H]1O)OCO5</smiles>

(69)<smiles>O[C@H]1C=C2CC[N+]3=Cc4cc5c(cc4[C@@H]([C@@H]1O)[C@H]23)OCO5</smiles>

(63) $R^{1}=R^{2}=R^{3}=H$

(72) $\mathrm{R}^{1}=\mathrm{H}, \mathrm{R}^{2}=\mathrm{OH}, \mathrm{R}^{3}=\mathrm{OMe}$

Lycorine-type<smiles>[R2]C1C=C2[C@@H]([R2])CN3Cc4cc5c(cc4[C@@H]1[C@H]2[C@H]3[R20])OCO5</smiles>

(78) $\mathrm{R}^{1}=\mathrm{C}(\mathrm{O}) \mathrm{CH}_{2} \mathrm{CH}(\mathrm{OH}) \mathrm{CH}_{3}, \mathrm{R}^{2}=\mathrm{OH}, \mathrm{R}^{3}=\mathrm{H}$

(80) $\mathrm{R}^{1}=\mathrm{H}, \mathrm{R}^{2}=\mathrm{OH}, \mathrm{R}^{3}=\mathrm{H}$<smiles>[R]C1c2cc3c(cc2[C@H]2[C@@H]4[C@@H](CCN14)C[C@@H](O)[C@@H]2O)OCO3</smiles>

(79) $\mathrm{R}=\mathrm{O}$<smiles></smiles>

(90)<smiles>O[C@H]1C[C@@H]2CC[N+]3=Cc4cc5c(cc4[C@@H]([C@@H]1O)[C@H]23)OCO5</smiles>

(74)<smiles>[R][R6](=O)c1cn2c3c(cccc13)-c1cc3c(cc1C2[R])OCO3</smiles><smiles>CO[C@H]1[C@@H](O)C2=CCN3Cc4cc5c(cc4[C@H](C23)[C@@H]1O)OCO5</smiles>

(91)<smiles>[R1]c1cc2c3c(c1)-c1cc(OC)c(OC)cc1C([R])N3CC2</smiles>

(62) $\mathrm{R}^{1}=\mathrm{R}^{2}=\mathrm{H}$

(87) $\mathrm{R}^{1}=\mathrm{OMe}, \mathrm{R}^{2}=\mathrm{O}$<smiles>[R20]Oc1cc2c(cc1O[R20])[C@@H]1[C@H]3C(=C[C@@H]([R2])[C@H]1[R20])CCN3C2</smiles>

(64) $\mathrm{R}^{1}=\mathrm{H}, \mathrm{R}^{2}=\mathrm{OMe}, \mathrm{R}^{3}=\mathrm{R}^{4}=\mathrm{Me}$ (73) $\mathrm{R}^{1}=\mathrm{H}, \mathrm{R}^{2}=\mathrm{OH}, \mathrm{R}^{3}=\mathrm{Me}, \mathrm{R}^{4}=\mathrm{H}$ (84) $R^{1}=R^{2}=R^{3}=H, R^{4}=M e$

(85) $R^{1}=R^{2}=H, R^{3}=R^{4}=M e$

(88) $\mathrm{R}^{1}=\mathrm{Ac}, \mathrm{R}^{2}=\mathrm{OH}, \mathrm{R}^{3}=\mathrm{H}, \mathrm{R}^{4}=\mathrm{Me}$<smiles>COc1cc2c(cc1OC)[C@H]1[C@H](O)[C@H](OC)[C@@H](O)C3=CCN(C2)[C@H]31</smiles>

(68)<smiles></smiles>

(65)<smiles>COc1cc2c(cc1OC)[C@H]1[C@@H]2[C@H](O)[C@H](OC)[C@@H](O)C2=CC[N+](C)(CCl)[C@@H]21</smiles>

(67)<smiles>COC1=C(OC)C=c2c3cc(OC)cc4c3c(c[n+]2CC4)=C1</smiles>

(89)

Figure 3. Amaryllidaceae alkaloids of homolycorine- and lycorine-type reported in Lycoris species. 


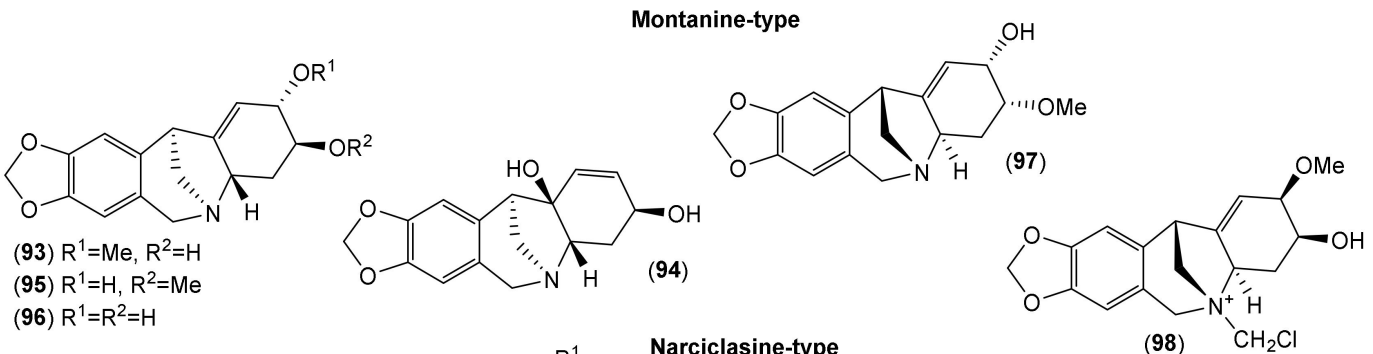<smiles>[R]c1c2c(cc3c1OCO3)C1=C[C@H](O)[C@@H](O)[C@H](O)[C@H]1NC2</smiles><smiles>[R1]c1ccc2c(c1)-c1cc3c(cc1C([R])N2[R])OCO3</smiles>

(98) $\mathrm{CH}_{2} \mathrm{Cl}$

(101) $\mathrm{R}^{1}=\mathrm{H}, \mathrm{R}^{2}=\mathrm{Me}, \mathrm{R}^{3}=\mathrm{H}$

(103) $R^{1}=H, R^{2}=M e, R^{3}=O$

(99) $\mathrm{R}=\mathrm{H}$

(104) $R^{1}=\mathrm{H}_{1} \mathrm{R}^{2}=\left(\mathrm{CH}_{2}\right)_{2} \mathrm{CH}(\mathrm{Me})_{2}, \mathrm{R}^{3}=\mathrm{O}$

(105) $R^{1}=R^{2}=H, R^{3}=O$

(106) $\mathrm{R}^{1}=\mathrm{OH}, \mathrm{R}^{2}=\mathrm{Me}, \mathrm{R}^{3}=\mathrm{H}$<smiles>C[n+]1cc2cc3c(cc2c2ccccc21)OCO3</smiles>

(102)<smiles>c1ccc2c(c1)ncc1cc3c(cc12)OCO3</smiles>

(107)<smiles>[R12]C[C@H]1OCc2cc3c(cc2[C@@]12C=C[C@H](O)C[C@H]2N(C)C)OCO3</smiles>

(108) $\mathrm{R}^{1}=\mathrm{Et}, \mathrm{R}^{2}=\mathrm{OH}$ (111) $\mathrm{R}^{1}=\mathrm{Me}, \mathrm{R}^{2}=\mathrm{OH}$<smiles></smiles>

(109)<smiles></smiles>

(110)
Tazettine-type<smiles>CO[C@H]1O[C@@H](C)[C@@]2(C=C[C@H](OC)[C@H]3CN(C)C[C@H]32)c2cc3c(cc21)OCO3</smiles><smiles>CO[C@H]1C=C[C@]23c4cc5c(cc4C(=O)O[C@H]2CN(C)[C@@H]3C1)OCO5</smiles>

(113)

Figure 4. Amaryllidaceae alkaloids of montanine-, narciclasine-, and tazettine-type reported in Lycoris species.<smiles></smiles>

(119)<smiles>OCc1ccc(-c2nccc3ccccc23)o1</smiles>

(120)<smiles>CCCCCCN[C@H]1CCc2cc(OC)c(OC)c(OC)c2-c2ccc(OC)c(=O)cc21</smiles><smiles></smiles>

(122) $\mathrm{R}^{1}=\mathrm{H}, \mathrm{R}^{2}=(\mathrm{CO}) \mathrm{OCH}_{3}$ (123) $\mathrm{R}^{1}=\mathrm{H}, \mathrm{R}^{2}=\mathrm{Me}$ (124) $R^{1}=R^{2}=M e$<smiles>COc1ccc2c(cc1O)c1c3c(cc4cc(O)c(C)cc2c41)OCO3</smiles><smiles>COC(=O)N1CCc2cc(O)c(OC)c3c2C1Cc1ccc(OC)c(O)c1-3</smiles><smiles>COC(=O)N1CCc2cc3c(c4c2-c2c(ccc(OC)c2OC)CC41)OCO3</smiles><smiles>COc1ccc2c(c1OC)-c1c(cc(O)c(OC)c1OC)CC2[N+](C)(C)[O-]</smiles>

Figure 5. Alkaloids of other structural types isolated from Lycoris plants.

The most studied Lycoris species, L. radiata, also called spider lily, is a horticultural plant widely distributed in the south of China, Vietnam, Korea, Nepal and Malaysia [14,15,27]. Altogether 79 Aas have been either identified or isolated from either the bulbs or flowers of this plant (Table 1). This species is rich in galanthamine-, haemanthidine-, homolycorine- and lycorine-type Aas. From the bulbs of L. radiata, the alkaloid colchicine (128) has recently been isolated; this is also derived from tyrosine, as are AAs, but it is metabolite typical of the genus Colchicum [28]. The question is whether it is a real product of the Lycoris plant, and not a product of some contamination with another plant, since this is 
the only report of this alkaloid-type within the whole Amaryllidaceae family. The same is also true for the reports of further alkaloids of other structural types (117-120 and 122-128; Table 1). The natural existence of an $\mathrm{N}$-chloromethyl moiety in galanthamine-, lycorine-, and montanine-type AAs, such as in $N$-chloromethylgalanthamine (12), N-chloromethyllycoramine (16), N-chloromethylungiminorine (66), N-chloromethylnarcissidin (67), and lycolongirine C (98) isolated from bulbs of L. longituba, L. radiata, and L. sprengeri must be reinvestigated, because when halogenated solvents are used during the isolation process, this can result in the formation of $N$-chloromethyl alkaloid derivative artifacts [29]. In the course of phytochemical study of the mentioned Lycoris species, dichloromethane has been used for extraction and column chromatography of the alkaloids, and this can be the explanation for the isolation of the mentioned compounds [30]. Either HPLC/MS or GC/MS analysis should be used for analysis of the alkaloidal extract before the separation process. Then, the presence of isotopes of ${ }^{35} \mathrm{Cl}$ and ${ }^{37} \mathrm{Cl}$ in the mass spectra of the separated compounds can indicate the natural presence of $\mathrm{N}$-chlormethylated derivatives. To avoid the possible formation of the mentioned artifacts, ethylacetate is recommended to be used for the preparation of the alkaloidal extract instead of halogenated solvents such as dichloromethan or chloroform. Another question is the natural presence of the butoxy-moiety at the C6 position in homolycorine-type compounds such as $2 \alpha$-hydroxy- $6-O-n$-butyloduline (38) and $O$ - $n$-butyllycorenine (50), both isolated from $L$. aurea in a study from 2014 , due to the reactivity of the carbonyl and hemiacetal groups [29]. The authors stated the use of $95 \%$ alcohol for the total extraction of fresh bulbs, but the exact type of alcohol was not specified at the beginning of the isolation process [31]. It can be assumed that butanol was used for this initial isolation step. To avoid discussion and speculation as to whether the isolated product is really a natural compound and not an isolation artefact, it is necessary to specify the solvents used within all steps of the phytochemical study.

Some authors also discuss the natural origin of the methoxy- and ethoxy-moieties in homolycorine-type AAs [29], when either methanol or ethanol are used during separation. It should also be noted that some AAs reported in the genus Lycoris, such as 1,2,11,12-tetradehydrogalanthamine (7), 3-hydroxylatifaliumin C (114), dihydrolatifaliumin C (115), and latifaliumin C (116) $[32,33]$ have been only tentatively identified on the basis of their EI mass spectra. Further isolation and spectroscopic studies of these compounds are necessary for their unambiguous identification and structure determination. A complete overview of AAs identified and isolated from Lycoris species is summarized in the following table (Table 1). 
Table 1. Alkaloids reported in the genus Lycoris.

\begin{tabular}{|c|c|c|c|c|c|c|c|c|c|c|c|c|c|c|}
\hline & 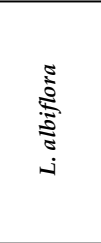 & 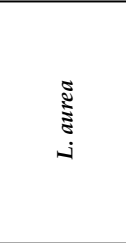 & 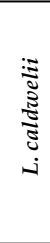 & 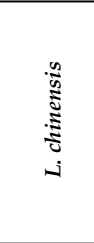 & 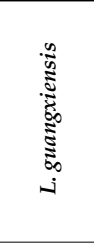 & 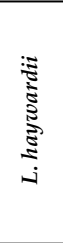 & 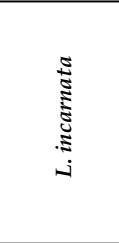 & 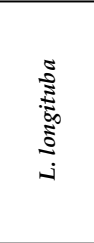 & 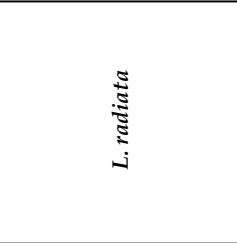 & 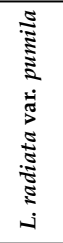 & 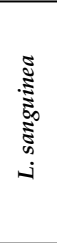 & 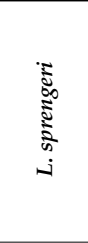 & 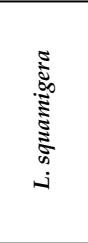 & 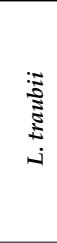 \\
\hline \multicolumn{15}{|l|}{ Belladine-type } \\
\hline $2 R$-Hydroxy- $\mathrm{N}, \mathrm{O}$-dimethylnorbelladine (1) & & & & & & & & & & & & & [34] & \\
\hline \multicolumn{15}{|l|}{ Crinine-type } \\
\hline Amabiline (2) & & & & & & & & & [28] & & & & & \\
\hline Ambelline (3) & & [32] & & & & & & & [32] & & & & & \\
\hline Crinine (4) & & & & & [35] & & & & & & & & & \\
\hline Crinamabine (5) & & & & & [32] & & & & [32] & & & & & \\
\hline Crinamidine (6) & & [32] & & & [32] & & & & [32] & & & & & \\
\hline \multicolumn{15}{|l|}{ Galanthamine-type } \\
\hline 1,2,11,12-Tetradehydrogalanthamine * (7) & & & & & & & & & [32] & & & & & \\
\hline 11ß-Hydroxygalanthamine (8) & & & & & & & & [30] & [28] & & & & & \\
\hline $\mathrm{N}$-Norgalanthamine/ $\mathrm{N}$-Demethylgalanthamine (9) & & {$[31,32]$} & & & {$[32,35]$} & & & [30] & [32] & & & & [34] & \\
\hline N-Allylnorgalanthamine (10) & & & & & [35] & & & & {$[28]$} & & & & & \\
\hline Galanthamine $N$-oxide (11) & & & & & & & [36] & & {$[37,38]$} & & & & & \\
\hline $\mathrm{N}$-(Chloromethyl)galanthamine (12) & & [31] & & & & & & [30] & & & & & & \\
\hline Galanthamine (13) & [39-41] & {$[32,41,42]$} & & {$[41,43]$} & {$[32,35]$} & [41] & {$[36,41,42]$} & {$[30,41]$} & {$[13,28,32,37,38,41,42]$} & [41] & & {$[41,44]$} & {$[34,41]$} & [45] \\
\hline O-Demethyllycoramine- $\mathrm{N}$-oxide (14) & & & & & & & & & {$[37]$} & & & & & \\
\hline O-Demethyllycoramine (15) & & & & & & & [36] & {$[30,41]$} & {$[37,38,46]$} & & & & [34] & \\
\hline $\mathrm{N}$-(Chloromethyl)lycoramine (16) & & [31] & & & & & & [30] & & & & & & \\
\hline Lycoramine- $\mathrm{N}$-oxide (17) & [39] & [32] & & & & & & & {$[32,37,38]$} & & & & & \\
\hline Lycoramine (18) & {$[39,41]$} & {$[32,41]$} & & {$[41,43]$} & {$[32,35]$} & [41] & {$[36,41]$} & [41] & {$[32,37,38,41]$} & [41] & & {$[41,44]$} & {$[34,41]$} & [45] \\
\hline Norlycoramine (19) & [41] & & & [41] & & & [41] & [41] & [41] & & & [41] & {$[41]$} & \\
\hline Narwedine (20) & [41] & [32] & & & {$[32,35]$} & & & [41] & & & & & [41] & \\
\hline Sanguinine (21) & & [41] & & [41] & & & [36] & {$[30,41]$} & {$[37,46]$} & & & & [34] & \\
\hline \multicolumn{15}{|l|}{ Galanthindole-type } \\
\hline Lycosinine B (22) & & & & & & & & & & & & [44] & & \\
\hline \multicolumn{15}{|l|}{ Haemanthamine-type } \\
\hline $3 \alpha$-Hydroxy-6 6 -acetylbulbispermine (23) & & & & & & & & & [47] & & & & & \\
\hline 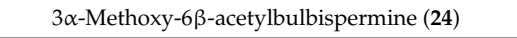 & & & & & & & & & [47] & & & & & \\
\hline $3 \alpha, 6 \beta$-Diacetylbulbispermine (25) & & & & & & & & & [47] & & & & & \\
\hline
\end{tabular}


Table 1. Cont.

\begin{tabular}{|c|c|c|c|c|c|c|c|c|c|c|c|c|c|c|}
\hline & 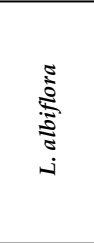 & 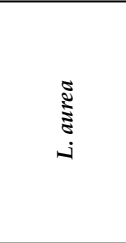 & 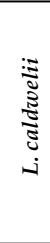 & 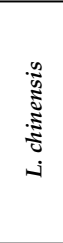 & 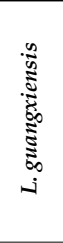 & 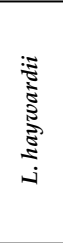 & 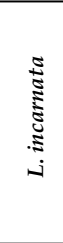 & 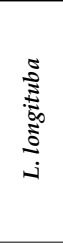 & 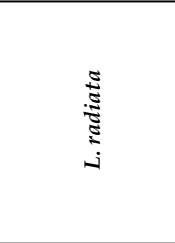 & 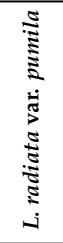 & 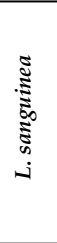 & 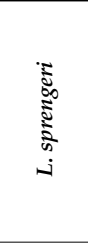 & 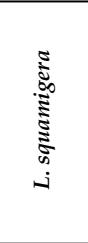 & 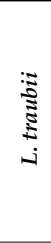 \\
\hline 6ß-Acetyl-8-hydroxy-9-methoxycrinamine (26) & & & & & & & & & [48] & & & & & \\
\hline 6-Hydroxycrinamine (27) & & & & & & & & & [13] & & & & & \\
\hline $6 \beta$-Acetoxycrinamine (28) & & & & & & & & & {$[13,48]$} & & & & & \\
\hline Haemanthamine (29) & [39] & {$[32,41]$} & & [41] & & & [41] & & {$[32,41]$} & & & {$[41,44]$} & [34] & \\
\hline Haemanthidine (30) & [39] & & & & & & [42] & [30] & [38] & & & [44] & {$[34,41]$} & \\
\hline 8-O-Demethylmaritidine (31) & & & & & & & & & {$[32,46]$} & & & & & \\
\hline 11-Hydroxyvittatine- $N$-oxide (32) & & [32] & & & & & & & & & & & & \\
\hline 11-Hydroxyvittatine (33) & & & & & & & & & [46] & & & & [34] & \\
\hline Vittatine (34) & & {$[31,32]$} & & & [32] & & & & {$[32,38]$} & & & & & \\
\hline \multicolumn{15}{|l|}{ Homolycorine-type } \\
\hline $2 \alpha$-Methoxy-6-O-ethyloduline (35) & & & & & & & & & {$[14,37]$} & & & & & \\
\hline $2 \alpha$-Methoxy-6-O-methyloduline (36) & & [49] & & & [32] & & & & [14] & & & & & \\
\hline $2 \alpha$-Hydroxy-6-O-methyloduline (37) & [39] & {$[31,32,49]$} & & & & & & & {$[13,32,37]$} & & & & & \\
\hline $2 \alpha$-Hydroxy-6-O-n-butyloduline (38) & & [31] & & & & & & & & & & & & \\
\hline $2 \alpha$-Hydroxyoduline (39) & & [49] & & & & & & & & & & & & \\
\hline Oduline (40) & & {$[32,49]$} & & & [32] & & & & {$[14,32]$} & & & & & \\
\hline $\begin{array}{l}2 \alpha \text {-Hydroxy-8-O-demethylhomolycorine- } N \text {-oxide } \\
(41)\end{array}$ & & & & & & & & & [48] & & & & & \\
\hline 8-O-Demethylhomolycorine- $N$-oxide (42) & & [50] & & & & & & & [48] & & & & & \\
\hline 8-O-Demethylhomolycorine (43) & & & & & & [41] & & & {$[14,38,41]$} & [41] & & & & \\
\hline 9-O-Demethylhomolycorine (44) & [39] & & & & & & & & {$[14,37]$} & & & & & \\
\hline 9-O-Demethyl-2 $\alpha$-hydroxyhomolycorine (45) & & & & & & & & & [37] & & & & & \\
\hline 8,9-Methylenedioxyhomolycorine- $\mathrm{N}$-oxide (46) & & & & & & & & & {$[32,47]$} & & & & & \\
\hline 8-O-Acetylhomolycorine- $\mathrm{N}$-oxide (47) & & & & & & & & & [13] & & & & & \\
\hline Homolycorine- $N$-oxide (48) & [39] & & & & & & & & {$[38,47]$} & & & & & \\
\hline Homolycorine (49) & {$[39,40]$} & {$[31,49]$} & & & & [41] & & & {$[13,14,38,41,42]$} & [41] & & [44] & & \\
\hline O-n-Butyllycorenine (50) & & [31] & & & & & & & & & & & & \\
\hline O-Ethyllycorenine (51) & & & & & & & & & {$[14,37]$} & & & & & \\
\hline O-Methyllycorenine (52) & & [31] & & & & & & & {$[14,37,38,41]$} & & & [44] & & \\
\hline O-Methyllycorenine- $N$-oxide (53) & & & & & & & & & [38] & & & & & \\
\hline $2 \alpha$-Methoxy-6-O-methyllycorenine (54) & & & & & & & & & & & & [44] & & \\
\hline
\end{tabular}


Table 1. Cont

\begin{tabular}{|c|c|c|c|c|c|c|c|c|c|c|c|c|c|c|}
\hline & 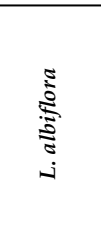 & 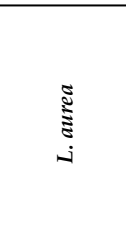 & 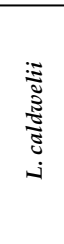 & 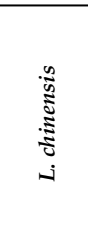 & 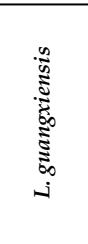 & 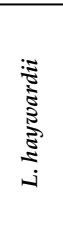 & 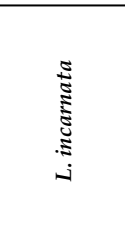 & 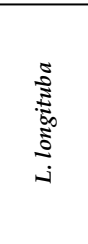 & 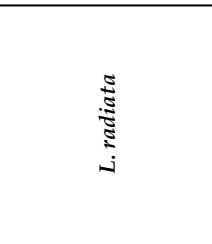 & 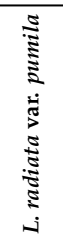 & 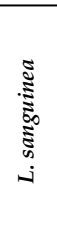 & 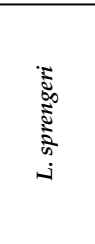 & 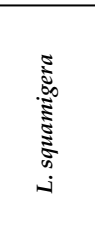 & 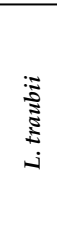 \\
\hline Lycorenine (55) & [40] & & & & & & & & {$[14,42]$} & & & [44] & & \\
\hline Radiatine (56) & & & & & & & & & [37] & & & & & \\
\hline Hippeastrine (57) & [39] & {$[31,32,49]$} & & & & & & & {$[13,14,32,37,38,41]$} & & & & & \\
\hline Hippeastrine- $\mathrm{N}$-oxide (58) & [39] & & & & & & & & [38] & & & & & \\
\hline Unsevine (59) & & & & & & & & & [32] & & & & & \\
\hline \multicolumn{15}{|l|}{ Hostasinine-type } \\
\hline Hostasinine A (60) & [39] & & & & & & & & & & & & & \\
\hline \multicolumn{15}{|l|}{ Ismine-type } \\
\hline Ismine (61) & & & & & & & & & & & & & [34] & \\
\hline \multicolumn{15}{|l|}{ Lycorine-type } \\
\hline Assoanine (62) & [41] & & & & & & & [41] & & & & & & \\
\hline Caranine (63) & [41] & [41] & & [41] & & & & [41] & [41] & [41] & & [41] & [41] & \\
\hline Galanthine (64) & [41] & [41] & & [41] & & [41] & {$[36,41]$} & [41] & [46] & & & {$[41,44]$} & [41] & \\
\hline Incartine (65) & [41] & [41] & & [41] & & [41] & {$[36,41]$} & [30] & & & & [41] & [41] & \\
\hline $\mathrm{N}$-(Chloromethyl)ungiminorine (66) & & & & & & & & & [37] & & & & & \\
\hline$N$-(Chloromethyl)narcissidine (67) & & & & & & & & [30] & & & & [44] & & \\
\hline Narcissidine (68) & & & & & & & & & & & & [44] & & \\
\hline (-)-epi-Zephyranthine (69) & & & & & & & & & [37] & & & & & \\
\hline Anhydrolycorine (70) & [41] & [41] & & [41] & & [41] & [41] & [41] & & [41] & & [41] & [41] & \\
\hline Dihydrolycorine (71) & & [32] & & & [32] & & & & {$[13,37]$} & & & & & \\
\hline 11-Methoxylycorine (72) & & & & & & & & & [37] & & & & & \\
\hline Pseudolycorine (73) & & & & & [35] & & & & {$[37,51]$} & & & & [34] & \\
\hline 5,6-Dehydrodihydrolycorine (74) & [41] & & & & & & & & [13] & & & & & \\
\hline 5,6-Dehydrolycorine (75) & & & & & & & & & {$[32,47]$} & & & & & \\
\hline 11,12-Didehydroanhydrolycorine (76) & & [41] & & [41] & & [41] & [41] & [41] & [41] & [41] & & [41] & [41] & \\
\hline 2-Hydroxyanhydrolycorine- $\mathrm{N}$-oxide (77) & & & [52] & & & & & & & & & & & \\
\hline 1-O-(3'-Hydroxybutanoyl)lycorine (78) & & & & & & & & & & & & & & [45] \\
\hline 6-Oxodihydrolycorine (79) & & & & & & & & & [13] & & & & & \\
\hline Lycorine (80) & [39-41] & $\begin{array}{c}{[31,32,41,} \\
42]\end{array}$ & & {$[41,43]$} & {$[32,35]$} & [41] & {$[36,41,42]$} & {$[30,41]$} & {$[13,28,32,38,41,42]$} & [41] & & {$[41,44]$} & {$[34,41]$} & [45] \\
\hline 1,2-Dihydroxy-anhydrolycorine- $N$-oxide (81) & & [50] & & & & & & & & & & & & \\
\hline 2-Hydroxy-6-oxoanhydrolycorine (82) & & [50] & & & & & & & & & & & & \\
\hline
\end{tabular}


Table 1. Cont

\begin{tabular}{|c|c|c|c|c|c|c|c|c|c|c|c|c|c|c|}
\hline & 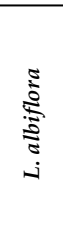 & تِ & 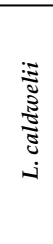 & 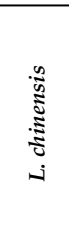 & 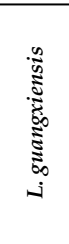 & 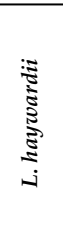 & 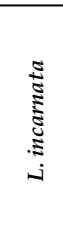 & 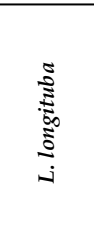 & 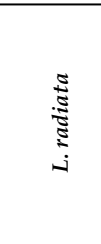 & 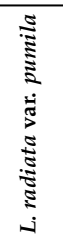 & 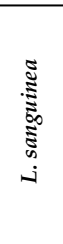 & 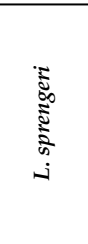 & 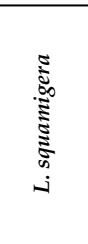 & 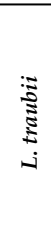 \\
\hline 1,2-Dihydroxy-6-oxoanhydrolycorine (83) & & [50] & & & & & & & & & & & & \\
\hline Norpluviine (84) & [41] & & & & & & & & & & & [41] & & \\
\hline Pluviine (85) & & {$[31,32]$} & & & [32] & [41] & & [41] & {$[32,37]$} & [41] & & [44] & [41] & \\
\hline Hippadine (86) & & & & & [32] & & & {$[30]$} & [37] & & & [44] & & \\
\hline Lycosprenine (87) & & & & & & & & & & & & [44] & & \\
\hline Sternbergine (88) & & & & & & & & & & & & & & [45] \\
\hline Tortuosine (89) & & & & & & & & & & & & [44] & & \\
\hline 1-Hydroxyungeremine $(\mathbf{9 0})$ & & & & & & & & & [48] & & & & & \\
\hline Ungiminorine (91) & & & & & & & [36] & & & & & & & [45] \\
\hline Ungiminorine- $N$-oxide (92) & & & & & & & [36] & & & & & & & \\
\hline \multicolumn{15}{|l|}{ Montanine-type } \\
\hline Montanine (93) & [41] & [41] & & [41] & & & & [41] & & & & & {$[34,41]$} & \\
\hline Pancratinine C/Squamigine (94) & & & & & & & & [41] & [37] & & & & [34] & \\
\hline (-)-3-O-Menthylpancracine (95) & & & & & & & & & [37] & & & & & \\
\hline Pancracine (96) & & & & & & & & & [37] & & & & & \\
\hline Montabuphine (97) & & & & & & & & & & & & [44] & & \\
\hline Lycolongirine C (98) & & & & & & & & [30] & & & & & & \\
\hline \multicolumn{15}{|l|}{ Narciclasine-type } \\
\hline 7-Deoxynarciclasine/Lycoricidine (99) & [39] & & & & & & & & [13] & & [53] & & [34] & [45] \\
\hline Narciclasine/Lycoricidinol (100) & [39] & & & & & & & & {$[16,37]$} & & [53] & & [34] & [45] \\
\hline 5,6-Dihydrobicolorine (101) & & & & & & & & & [13] & & & [44] & & \\
\hline Bicolorine (102) & & & & & & & & & & & [46] & & & \\
\hline $\mathrm{N}$-Methylcrinasiadine (103) & & & & & & & & [30] & & & & & & \\
\hline $\mathrm{N}$-Isopentylcrinasiadine (104) & & & & & & & & & & & & [44] & & \\
\hline Crinasiadine (105) & & & & & & & & & & & & [44] & & \\
\hline $\begin{array}{l}\text { 5,6-Dihydro-5-methyl-2-hydroxyphenanthridine } \\
(\mathbf{1 0 6})\end{array}$ & & {$[50]$} & & & & & & & [47] & & & & & \\
\hline Trisphaeridine (107) & & & & & & & & {$[30,41]$} & [46] & & & {$[41,44]$} & & \\
\hline \multicolumn{15}{|l|}{ Tazettine-type } \\
\hline 3-O-Ethyltazettinol (108) & & [54] & & & & & & & & & & & & \\
\hline Deoxydihydrotazettine (109) & & & & & & & & & [28] & & & & & \\
\hline Deoxypretazettine (110) & & & & & & & & {$[30,41]$} & {$[28,41]$} & & & & & \\
\hline
\end{tabular}


Table 1. Cont.

\begin{tabular}{|c|c|c|c|c|c|c|c|c|c|c|c|c|c|c|}
\hline & \begin{tabular}{l}
5 \\
\multirow{5}{5}{} \\
\multirow{3}{5}{} \\
i
\end{tabular} & స్ & 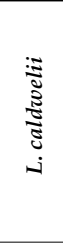 & 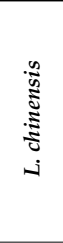 & 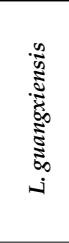 & 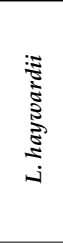 & 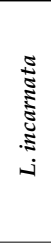 & 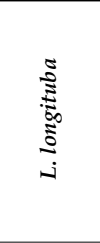 & 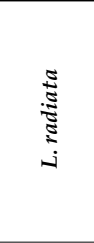 & 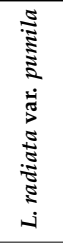 & 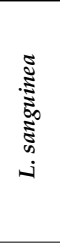 & 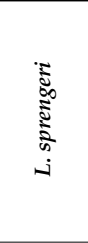 & 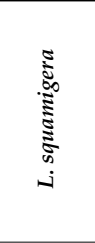 & 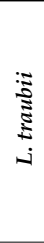 \\
\hline Tazettine (111) & [41] & [41] & & [41] & & [41] & & {$[30,41]$} & {$[38,41]$} & [41] & & {$[41,44]$} & {$[34,41]$} & \\
\hline 6-O-Methylpretazettine (112) & & & & & & & & & & & & & [34] & \\
\hline 3-Epimacronine (113) & & & & & & & & & [28] & & & & & \\
\hline 3-Hydroxylatifaliumin C* (114) & & & & & [32] & & & & [32] & & & & & \\
\hline Dihydrolatifaliumin C* (115) & & [32] & & & [32] & & & & {$[32]$} & & & & & \\
\hline Latifaliumin $C^{*}(\mathbf{1 1 6})$ & & [32] & & & & & & & & & & & & \\
\hline \multicolumn{15}{|l|}{ Other structural types } \\
\hline Norharmane (117) & & & & & & & & {$[30]$} & & & & & & \\
\hline Harmane (118) & & & & & & & & {$[30]$} & & & & & & \\
\hline Lycolongirine A (119) & & & & & & & & [30] & & & & & & \\
\hline Perlolyrine (120) & & & & & & & & [30] & & & & & & \\
\hline Colchicine (121) & & & & & & & & & [28] & & & & & \\
\hline $\mathrm{N}$-Methoxycarbonyl-2-demethylisocorydione (122) & & & & & & & & & [48] & & & & & \\
\hline 2-Demethylisocorydione (123) & & {$[50]$} & & & & & & & & & & & & \\
\hline Isocorydione (124) & & {$[50]$} & & & & & & & & & & & & \\
\hline 8-Demethyldehydrocrebanine (125) & & {$[50]$} & & & & & & & & & & & & \\
\hline N-Methoxycarbonyllindcarpine (126) & & & [52] & & & & & & & & & & & \\
\hline N-Methoxycarbonylnandigerine (127) & & & [52] & & & & & & & & & & & \\
\hline 10-O-Methylhernovine- $\mathrm{N}$-oxide (128) & & & [52] & & & & & & & & & & & \\
\hline
\end{tabular}




\section{Studied Biological Activities of Extracts and Alkaloids Isolated from Lycoris Species}

\subsection{Antitumor Activity}

In recent decades, AAs such as lycorine, pancratistatine, narciclassine, haemanthamine, and montanine have been intensively studied for their cytotoxic potential. All alkaloids displayed multiple properties towards various cancer cell lines including MOLT-4, HepG2, HeLa, MCF-7, CEM, K562, A549, Caco-2, HT-29, A2780 and others [55-62]. Thus, extracts and alkaloids isolated from different Lycoris species have been primarily tested for their cytotoxic activity on different cancerous cells. The dichloromethane extract (DCME) of bulbs of L. aurea, when tested both in vivo and in vitro against the murine sarcoma 180 cell line, using MTT assay [49], demonstrated promising inhibition effects on the cells in a dose-dependent manner. The in vivo study using sarcoma 180 bearing mice demonstrated inhibitory rates of $27.9 \%$ for a dosage of $20 \mu \mathrm{g} / \mathrm{mL}$ of DCME, 37.2\% for a dosage of $40 \mu \mathrm{g} / \mathrm{mL}$, and $53.5 \%$ for a dosage of $120 \mu \mathrm{g} / \mathrm{mL}$ [49].

Crude extract, DCME extract and pure alkaloids isolated from bulbs of L. aurea were also evaluated for their antiproliferative activities against the SH-SY5Y cell line. The crude and DCME extracts revealed cytotoxicity at concentrations of $5 \mu \mathrm{g} / \mathrm{mL}$ [31]; of the pure alkaloids, only lycorine (80) demonstrated significant cytotoxicity at a concentration $6.25 \mu \mathrm{M}$ [31].

Alkaloidal extracts of three Lycoris species were screened for their cytotoxic potential against HepG2 cells at a concentration of $10 \mu \mathrm{g} / \mathrm{mL}$ with inhibitory rates of $78.0 \%, 84.9 \%$, and $66.8 \%$ for $L$. aurea, L. radiata and L. guangxiensis, respectively [32].

An alcoholic extract of fresh bulbs of L. albiflora showed promising cytotoxic activity against HL-60 cells, with an $\mathrm{IC}_{50}$ value of $1.7 \mu \mathrm{g} / \mathrm{mL}$ [39]. This resulted in a detailed phytochemical study being undertaken to isolate pure AAs and test them for their cytotoxic activity; fifteen AAs were isolated (Table 1), which were tested for their cytotoxic activity against the cancerous cell line HL-60. The most potent AAs were also subjected to a cytotoxic screening against HSC-2 cells [39]. Narciclassine-type alkaloids 7-deoxynarciclasine (99; also known as lycoricidine) and narciclasine (100, also known as lycoricidinol) induced apoptosis in both HL-60 and HSC-2 cells. Moreover, narciclasine (100) induced transient autophagy and morphological changes in mitochondria in the early stages of the apoptotic cell death process in HSC-2 cells [39]. Within previous studies, narciclasine (100) exhibited potent in vitro cytotoxicity against various cancer cells and showed great potential against primary brain cancers, as well as brain metastases in vivo [63-65]. Within the latest study, narciclasine (100) displayed preferential cytotoxicity towards primary effusion lymphoma cell lines (PEL), an aggressive type of non-Hodgkin lymphoma, with $\mathrm{IC}_{50}$ values ranging from 7 to $14 \mathrm{nM}$ [66]. 7-Deoxynarciclasine (99) displayed approximately 10 times lower cytotoxicity against the tested PEL cell lines $\left(\mathrm{IC}_{50}=82-162\right.$ nM) [66]. Previous in vitro studies frequently focused on the cytotoxicity of narciclasine against fibroblast $\left(\mathrm{IC}_{50}=7.5 \mu \mathrm{M}\right)$ and cancer cells $\left(\mathrm{IC}_{50}=30 \mathrm{nM}\right)$, which indicated the compound's selectivity to cancer cells and only higher concentrations affected the viability of fibroblasts [67]. On the other hand, it was reported that narciclasine (100) showed only modest anti-tumor effects in mice in vivo, with considerable toxicity [68]. Thus, narciclasine (100) has not been tested in human clinical trials up to now. The inhibitory effects on L02 (human normal liver cell line) and murine macrophages RAW264.7 indicated that narciclasine (100) might have significant side effects, and, therefore, further studies are urgently needed [16]. Narciclasine (100) has also been shown to inhibit the cytotoxicity of calprotectin in rat adjuvant arthritis mode, and several studies have reported that narciclasine (100) exhibits strong anti-inflammation activity in vitro and in vivo [69]. LPS-stimulated RAW264.7 cells were employed to investigate the anti-inflammatory effects of narciclasine (100) in order to explore its underlying mechanism [16]. Recently, narciclasine was named 'Molecule of the Week' by the American Chemical Society (ACS) for its potential as a cancer drug [66].

The in vitro antiproliferation assay of hippeastrine (57) isolated from fresh bulbs of L. radiata revealed strong inhibition of proliferation of HT-29 and Hep G2 cells in an intuitive dose-dependent 
manner, with $\mathrm{IC}_{50}$ values of $3.98 \pm 0.29 \mu \mathrm{g} / \mathrm{mL}$ and $11.85 \pm 0.20 \mu \mathrm{g} / \mathrm{mL}$, respectively [12]. The results of the cytotoxic studies of AAs isolated from Lycoris species are summarized in the following table (Table 2).

Table 2. Impact of Amaryllidaceae alkaloids (AA) isolated from Lycoris plants on proliferation of cancer and non-cancer cells using in vitro assays. Results are expressed as $\mathrm{IC}_{50}$ values in $\mu \mathrm{M}$, unless otherwise stated.

\begin{tabular}{|c|c|c|c|c|c|c|c|c|c|c|c|}
\hline \multirow[b]{2}{*}{ Alkaloid (No.) } & \multicolumn{10}{|c|}{ Cell Line } & \multirow[b]{2}{*}{ Ref. } \\
\hline & HL-60 & A549 & $\begin{array}{l}\text { MC } \\
\text { F-7 }\end{array}$ & $\begin{array}{l}\text { BEN- } \\
\text { MEN-1 }\end{array}$ & $\begin{array}{l}\text { CCF- } \\
\text { STTG1 }\end{array}$ & $\begin{array}{l}\mathrm{CH} \\
\mathrm{G}-5\end{array}$ & $\begin{array}{c}\text { SH } \\
\text { G-44 }\end{array}$ & U251 & $\begin{array}{l}\text { SMM } \\
\text { C-7721 }\end{array}$ & W480 & \\
\hline 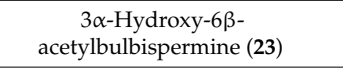 & $\begin{array}{c}7.1 \pm \\
0.9\end{array}$ & & & $>100$ & $\begin{array}{c}29.4 \pm \\
4.1\end{array}$ & $\begin{array}{c}29.4 \pm \\
5.3\end{array}$ & $\begin{array}{c}28.3 \pm \\
2.7\end{array}$ & $\begin{array}{c}15.8 \pm \\
1.7\end{array}$ & $\begin{array}{l}66.8 \pm \\
9.4\end{array}$ & $\begin{array}{l}53.5 \pm \\
12.4\end{array}$ & [47] \\
\hline 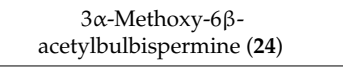 & $\begin{array}{c}8.6 \pm \\
1.4\end{array}$ & & & $>100$ & $\begin{array}{c}29.7 \pm \\
5.4\end{array}$ & $\begin{array}{c}29.6 \pm \\
6.3\end{array}$ & $\begin{array}{c}29.1 \pm \\
3.8\end{array}$ & $\begin{array}{c}16.7 \pm \\
2.6\end{array}$ & $\begin{array}{c}68.2 \pm \\
12.3\end{array}$ & $\begin{array}{l}50.1 \pm \\
7.8\end{array}$ & [47] \\
\hline $\begin{array}{c}3 \alpha, 6 \beta \text {-Diacetylbulbispermine } \\
\text { (25) }\end{array}$ & $\begin{array}{c}7.3 \pm \\
1.1\end{array}$ & & & $>100$ & $\begin{array}{c}27.1 \pm \\
5.1\end{array}$ & $\begin{array}{c}30.1 \pm \\
4.4\end{array}$ & $\begin{array}{c}27.1 \pm \\
3.2\end{array}$ & $\begin{array}{c}17.4 \pm \\
2.1\end{array}$ & $\begin{array}{c}63.2 \pm \\
11.8\end{array}$ & $\begin{array}{c}51.1 \pm \\
10.9\end{array}$ & [47] \\
\hline $\begin{array}{l}\text { 6ß-Acetyl-8-hydroxy-9- } \\
\text { methoxycrinamine (26) }\end{array}$ & $\begin{array}{c}8.6 \pm \\
1.4\end{array}$ & & & $>100$ & $\begin{array}{c}29.4 \pm \\
4.1\end{array}$ & $\begin{array}{c}29.6 \pm \\
5.3\end{array}$ & $\begin{array}{c}27.1 \pm \\
3.2\end{array}$ & $\begin{array}{c}17.4 \pm \\
2.1\end{array}$ & $\begin{array}{c}68.2 \pm \\
12.3\end{array}$ & $\begin{array}{l}53.5 \pm \\
12.4\end{array}$ & [48] \\
\hline $6 \beta$-Acetoxycrinamine (28) & 8.1 & 24.3 & 15.0 & & & & & & & & [13] \\
\hline $\begin{array}{l}\text { 8,9-Methylenedioxyhomolycorine- } \\
\text { N-oxide (46) }\end{array}$ & $>100$ & & & $>100$ & $\begin{array}{c}83.2 \pm \\
13.7\end{array}$ & $>100$ & $>100$ & $>100$ & $\begin{array}{c}86.2 \pm \\
17.4\end{array}$ & $>100$ & [47] \\
\hline $\begin{array}{l}\text { 8-O-Acetylhomolycorine- } \\
\text { N-oxide (47) }\end{array}$ & $>40$ & $>40$ & $>40$ & & & & & & & & \\
\hline Homolycorine- $N$-oxide (48) & $>100$ & & & $>100$ & $>100$ & $\begin{array}{c}93.0 \pm \\
21.1\end{array}$ & $>100$ & $>100$ & $\begin{array}{c}85.0 \pm \\
16.2\end{array}$ & $>100$ & [47] \\
\hline $\begin{array}{l}\text { 5,6-Dehydrodihydrolycori- } \\
\text { ne (79) }\end{array}$ & $>40$ & $>40$ & $>40$ & & & & & & & & [13] \\
\hline 5,6-Dehydrolycorine (75) & $\begin{array}{c}10.8 \pm \\
1.6\end{array}$ & & & $>100$ & $\begin{array}{c}10.3 \pm \\
0.9\end{array}$ & $\begin{array}{c}10.2 \pm \\
1.6\end{array}$ & $\begin{array}{c}9.4 \pm \\
1.3\end{array}$ & $\begin{array}{c}11.8 \pm \\
0.7\end{array}$ & $\begin{array}{c}10.5 \pm \\
0.9\end{array}$ & $\begin{array}{c}11.6 \pm \\
1.1\end{array}$ & [47] \\
\hline $\begin{array}{l}\text { 2-Hydroxy-anhydrolycorine- } \\
N \text {-oxide (77) }\end{array}$ & & & & $>100$ & $>100$ & $>100$ & $>100$ & 93.7 & & & [52] \\
\hline 1-Hydroxyungeremine (90) & $\begin{array}{c}10.8 \pm \\
1.6\end{array}$ & & & $>100$ & $\begin{array}{c}10.3 \pm \\
0.9\end{array}$ & $\begin{array}{c}10.2 \pm \\
1.6\end{array}$ & $\begin{array}{c}9.4 \pm \\
1.3\end{array}$ & $\begin{array}{c}11.8 \pm \\
0.9\end{array}$ & $\begin{array}{c}10.5 \pm \\
0.9\end{array}$ & $\begin{array}{c}11.6 \pm \\
1.1\end{array}$ & [48] \\
\hline $\begin{array}{l}\text { 7-Deoxynarciclasine/Lyco- } \\
\text { ricidine (99) }\end{array}$ & 0.15 & & & & & & & & & & [39] \\
\hline $\begin{array}{c}\text { Narciclasine/Lycoricidi-nol } \\
\text { (100) }\end{array}$ & 0.018 & & & & & & & & & & [39] \\
\hline $\begin{array}{c}\text { 5,6,-Dihydro-5-methyl- } \\
\text { 2-hydroxyphenanthridine (106) }\end{array}$ & $\begin{array}{c}81.3 \pm \\
15.7\end{array}$ & & & $>100$ & $>100$ & $>100$ & $>100$ & $>100$ & $>100$ & $>100$ & [47] \\
\hline Alkaloid (No.) & HT-29 & $\begin{array}{l}\text { Hep } \\
\text { G-2 }\end{array}$ & $\begin{array}{l}\text { SK- } \\
\text { OV-3 }\end{array}$ & SCL-1 & $\begin{array}{c}\text { CA } \\
\text { L-27 }\end{array}$ & $\begin{array}{c}\text { UMSC } \\
\text { C-1 }\end{array}$ & $\begin{array}{l}\text { Detroi } \\
\mathrm{t}-562\end{array}$ & $\begin{array}{l}\text { SCC- } \\
\text { PKU }\end{array}$ & $\begin{array}{c}\text { TC } \\
\text { A-83 }\end{array}$ & $\begin{array}{l}\mathrm{HS} \\
\mathrm{C}-2\end{array}$ & Ref. \\
\hline $\begin{array}{l}\text { 8-O-Demethylhomolycorine- } \\
\text { N-oxide (42) }\end{array}$ & & 11.6 & & 13.2 & 12.3 & 12.3 & 12.9 & 13.2 & 16.7 & & {$[50]$} \\
\hline Hippeastrine (57) & $\begin{array}{c}12.6 \pm \\
0.92\end{array}$ & $\begin{array}{l}37.62 \\
\pm 0.63\end{array}$ & & & & & & & & & [12] \\
\hline $\begin{array}{c}\text { 2-Hydroxy-anhydrolycorine- } \\
N \text {-oxide (77) }\end{array}$ & & 67.7 & 76.2 & & & & & & & & [52] \\
\hline Alkaloid (No.) & HT-29 & $\begin{array}{l}\text { Hep } \\
\text { G-2 }\end{array}$ & $\begin{array}{l}\text { SK- } \\
\text { OV-3 }\end{array}$ & $\begin{array}{l}\text { SC } \\
\text { L-1 }\end{array}$ & $\begin{array}{c}\text { CA } \\
\text { L-27 }\end{array}$ & $\begin{array}{l}\text { UMSC } \\
\text { C-1 }\end{array}$ & $\begin{array}{c}\text { Detroi } \\
\mathrm{t}-562\end{array}$ & $\begin{array}{l}\text { SCC- } \\
\text { PKU }\end{array}$ & $\begin{array}{c}\text { TC } \\
\text { A-83 }\end{array}$ & $\begin{array}{l}\text { HS } \\
\text { C-2 }\end{array}$ & Ref. \\
\hline $\begin{array}{l}\text { 1,2-Dihydroxy-anhydrolycorine- } \\
\text { N-oxide (81) }\end{array}$ & & $>100$ & & $>100$ & $>100$ & $>100$ & 94.3 & $>100$ & $>100$ & $>100$ & [50] \\
\hline $\begin{array}{c}\text { 2-Hydroxy-6-oxoanhydrolycorine } \\
(\mathbf{8 2})\end{array}$ & & $>100$ & & $>100$ & $>100$ & $>100$ & $>100$ & 95.5 & 91.2 & & [50] \\
\hline $\begin{array}{l}\text { 1,2-Dihydroxy-6- } \\
\text { oxoanhydrolycorine (83) }\end{array}$ & & $>100$ & & $>100$ & $>100$ & $>100$ & $>100$ & 88.3 & 91.8 & & [50] \\
\hline $\begin{array}{l}\text { 7-Deoxynarciclasine/Lyco- } \\
\text { ricidine (99) }\end{array}$ & & & & & & & & & & $\begin{array}{c}1.7 \pm \\
0.2\end{array}$ & [39] \\
\hline $\begin{array}{c}\text { Narciclasine/Lycoricidi-nol } \\
\text { (100) }\end{array}$ & 1.373 & 0.08 & & & & & & & & 0.05 & $\begin{array}{l}{[16,} \\
39]\end{array}$ \\
\hline $\begin{array}{c}\text { 5,6,-Dihydro-5-methyl-2- } \\
\text { hydroxyphenanthridine (106) }\end{array}$ & & $>100$ & & $>100$ & $>100$ & $>100$ & $>100$ & 87.6 & $>100$ & & [50] \\
\hline
\end{tabular}




\subsection{Biological Activity Connected with Alzheimer's Disease}

$\mathrm{AD}$ is a major neurodegenerative illness, and is the major cause of dementia associated with aging [70]. The current treatment of $\mathrm{AD}$ is only symptomatic and mainly involves restoring of acetylcholine (ACh) levels through acetylcholinesterase (AChE) inhibition [71]. Three AChE inhibitors, namely donepezil, galanthamine (13) and rivastigmine, are currently used as the main therapeutic option for AD treatment [72]. Since the Amaryllidaceae alkaloid galanthamine (13) has been introduced into clinical practice, other Amaryllidaceae alkaloids have received attention as potential AChE inhibitors [5,73-76]. Surprisingly, only a few AAs isolated from Lycoris plants have been studied in terms of their AChE inhibition potential. AAs isolated from bulbs of L. longituba (Table 1) have been screened for their AChE inhibition effects. The most active alkaloids in the AChE assay belong to the galanthamine structural type of AAs, which was in agreement with previous results. The best AChE inhibition activity has been obtained for galanthamine $\left(\mathrm{IC}_{50}=2.43 \pm 0.66 \mu \mathrm{M}\right)$, which is already used in the therapy of AD as a competitive, reversible, selective inhibitor of AChE. Promising AChE inhibition activities were obtained also for further AAs of galanthamine-type (Table 3), and, interestingly, for $N$-methylcrinasiadine $\left(\mathbf{1 0 3} ; \mathrm{IC}_{50}=4.23 \pm 1.13 \mu \mathrm{M}\right)$ and deoxypretazettine (110; $\left.\mathrm{IC}_{50}=8.44 \pm 0.83 \mu \mathrm{M}\right)$. Moreover, isolated AAs were tested for their neuroprotective effects against $\mathrm{CoCl}_{2}, \mathrm{H}_{2} \mathrm{O}_{2}$ and $\mathrm{A} \beta_{25-35}$-induced neuronal cell death in dopaminergic neuroblastoma SH-SY5Y cells [30]. Incartine (65), trisphaeridine (107), N-(chloromethyl)galanthamine (12), sanquinine (21), O-demethyllycoramine (15), and deoxypretazettine (110) showed significant neuroprotective effects against all three injury models [30]. Lycolongirine C (103) and N-methylcrinasiadine (110) exhibited significant neuroprotective activities against $\mathrm{H}_{2} \mathrm{O}_{2}$ and $\mathrm{A} \beta_{25-35}$-induced cell death [30].

Table 3. AChE inhibitory activity of Amaryllidaceae alkaloids isolated from bulbs of L. longituba. [30].

\begin{tabular}{ccc}
\hline Alkaloid (No.) & \% AChE Inhibition $\mathbf{( 1 0 0 ~} \boldsymbol{\mu M})$ & IC $_{\mathbf{5 0}}(\boldsymbol{\mu M})$ \\
\hline 11ß-Hydroxygalanthamine (8) & $96 \pm 0$ & $3.04 \pm 0.61$ \\
N-Norgalanthamine (9) & $92 \pm 1$ & $2.76 \pm 0.65$ \\
N-(Chloromethyl)galanthamine (12) & $94 \pm 1$ & $5.55 \pm 0.63$ \\
Galanthamine (13) & $95 \pm 1$ & $2.43 \pm 0.66$ \\
O-Demethyllycoramine (15) & $86 \pm 1$ & $8.13 \pm 1.49$ \\
N-(Chloromethyl)lycoramine (16) & $79 \pm 1$ & $25.76 \pm 1.09$ \\
Sanguinine (21) & $93 \pm 0$ & $5.30 \pm 0.76$ \\
Haemanthidine (30) & $39 \pm 1$ & $208.10 \pm 1.58$ \\
Incartine (65) & $41 \pm 1$ & $148.70 \pm 1.46$ \\
N-(Chloromethyl)narcissidine (67) & $36 \pm 3$ & $190.70 \pm 2.00$ \\
Lycorine (80) & $32 \pm 2$ & $224.80 \pm 3.01$ \\
Hippadine (86) & $42 \pm 2$ & $117.60 \pm 1.79$ \\
Lycolongirine C (98) & $45 \pm 2$ & $194.80 \pm 2.31$ \\
N-Methylcrinasiadine (103) & $85 \pm 1$ & $4.23 \pm 1.13$ \\
Trisphaeridine (107) & $33 \pm 1$ & $190.70 \pm 2.00$ \\
Deoxypretazettine (110) & $89 \pm 0.38$ & $8.44 \pm 0.83$ \\
\hline
\end{tabular}

The crude and DCME extracts obtained from the bulbs of L. aurea were also tested for their neuroprotective effects against $\mathrm{CoCl}_{2}$, and $\mathrm{H}_{2} \mathrm{O}_{2}$-induced cell injuries in SH-SY5Y cells [31]. Both extracts exhibited modest neuroprotective effects against $\mathrm{CoCl}_{2}$-induced SH-SY5Ycell injury, but a significant effect in $\mathrm{H}_{2} \mathrm{O}_{2}$-induced SH-SY5Y cell injury [31]. The mentioned extracts were further subjected to column chromatography and separation processes, resulting in the isolation of thirteen AAs (Table 1), which were also tested for their neuroprotective effects. Alkaloids belonging to the homolycorine- and galanthamine-type $(9,12,13,16,37,38,50$, and 52) exhibited significant neuroprotective effects against $\mathrm{CoCl}_{2}$-induced $\mathrm{SH}$-SY5Y cell injury, while alkaloids 9, 12, 16, 37, 38, 50, 53, and 85 showed obvious neuroprotective effects against $\mathrm{H}_{2} \mathrm{O}_{2}$-induced SH-SY5Y cell death [31].

Seven AAs isolated from L. sprengeri were evaluated for their neuroprotective activities against the same models [44]. O-methyllycorenine (52), and hippadine (86) exhibited significant neuroprotective 
effects against $\mathrm{H}_{2} \mathrm{O}_{2}$-induced SH-SY5Y cell death; lycosprenine (87), O-methyllycorenine (52), and tortuosine (89) showed obvious neuroprotective effects against $\mathrm{CoCl}_{2}$-induced SH-SY5Y cell injury [44]. The results obtained within the mentioned studies indicate that compounds of the same structural type of AAs (e.g., homolycorine, lycorine and galanthamine) may have potential for further development as neuroprotective compounds.

\subsection{Antimalarial Activity}

Malaria is one of the most common vector-borne infectious diseases. This disease is caused by protozoan parasites of the genus Plasmodium [77]. Alkaloids isolated from L. radiata were evaluated in vitro for their antimalarial activity using the drug-resistant D-6 strain and drug sensitive W-2 strain of P. falciparum [47]. Within the tested AAs, only 5,6-dehydrolycorine (75) exhibited antimalarial activity, with $\mathrm{IC}_{50}$ values of $2.3 \mu \mathrm{M}$ for the D-6 strain and $1.9 \mu \mathrm{M}$ for the $\mathrm{W}-2$ strain of P. falciparum [47]. Other studied AAs displayed only weak or no antimalarial activity against the studied strains (Table 4).

Table 4. In vitro antimalarial activity of Amaryllidaceae alkaloids isolated from L. radiata against two Plasmodium falciparum strains [47].

\begin{tabular}{|c|c|c|}
\hline Alkaloid (No.) & D-6 IC $50(\mu \mathrm{M})$ & $\mathrm{W}-2 \mathrm{IC}_{50}(\mu \mathrm{M})$ \\
\hline $3 \alpha$-Hydroxy- $6 \beta$-acetylbulbispermine (23) & 17.9 & 19.3 \\
\hline $3 \alpha$-Methoxy- $6 \beta$-acetylbulbispermine (24) & 21.3 & 23.4 \\
\hline $3 \alpha, 6 \beta$-Diacetylbulbispermine (25) & 18.9 & 20.1 \\
\hline 8,9-Methylenedioxyhomolycorine- $N$-oxide (46) & $>100$ & $>100$ \\
\hline Homolycorine- $N$-oxide (48) & $>100$ & $>100$ \\
\hline 5,6-Dehydrolycorine (75) & 2.3 & 1.9 \\
\hline 5,6,-Dihydro-5-methyl-2-hydroxyphenanthridine (106) & $>100$ & $>100$ \\
\hline Chloroquine * & $9.8^{\mathrm{a}}$ & $6.7^{\mathrm{a}}$ \\
\hline
\end{tabular}

${ }^{\mathrm{a}}$ Chloroquine data are expressed as $\mathrm{IC}_{50}$ values in $\mathrm{nM} ;{ }^{*}$ standard.

\subsection{Further Studied Biological Activities}

Alkaloids $2 \alpha$-methoxy-6-O-ethyloduline (35), $2 \alpha$-methoxy-6-O-methyloduline (36) and hippeastrine (57), all isolated from the bulbs of Lycoris radiata, showed weak antiviral activities against flu virus $A$ with $\mathrm{IC}_{50}$ values of $2.06,0.69$ and $2.71 \mu \mathrm{g} \cdot \mathrm{mL}^{-1}$ and $\mathrm{CC}_{50}$ values of $14.37,4.79$, and $80.12 \mu \mathrm{g} . \mathrm{mL}^{-1}$ [14], respectively.

Aphids are one of the most destructive and economically important pests of plants on earth and extensive use of insecticides has resulted in the development of insecticide resistance among aphids across regions [78]. Thus, the insecticidal activity of ten AAs isolated from L. radiata against Aphis citricola has been studied [28]. $\mathrm{LD}_{50}$ values were measured by a capillary drip method and nine of the tested AASs displayed aphicial activity. N-Allylnorgalanthamine (10) possessed the highest aphicial activity $\left(\mathrm{LD}_{50}=4.92 \pm 0.83 \mathrm{ng} / \mathrm{aphid}\right)$, which was comparable with the commercial pesticide methomyl $\left(\mathrm{LD}_{50}=2.91 \pm 0.18 \mathrm{ng} / \mathrm{aphid}\right)$. Inhibition of $\mathrm{AChE}$ is a main target enzyme for many insecticides (especially carbamates and organophosphates) [79]; the in vitro inhibition of AChE of $\mathrm{N}$-allylnorgalanthamine (10) has also been studied. This compound demonstrated remarkable inhibition activity against $\mathrm{AChE}$ in $A$. citricola with a value of $\mathrm{IC}_{50}=2.1 \mathrm{nM}$.

\section{Conclusions}

In conclusion, this review summarizes the ethnobotanical, phytochemical, and pharmacological information about plants and AAs of the genus Lycoris. So far, fourteen Lycoris species have been phytochemically studied, and nearly 120 AAs of different structural types have been either identified or isolated. The presence and structures of some reported AAs must be reevaluated, as they may be isolation artifacts, and not naturally occurring compounds. Lycoris plants are above all a rich source of homolycorine- and lycorine-type AAs. Most of the isolated AAs have been studied for different 
biological activities with impact on antitumor and neuroprotective activities. The antimalarial, antiviral and insecticidal activities of some AAs have also been described. In the light of the presented overview of scientific data, the genus Lycoris can be recognized as an interesting source of different structural types of AAs with a wide range of biological activities.

Author Contributions: Conceptualization, L.C.; Methodology, L.C. and K.B., Formal analysis, K.B., and L.O.; Investigation, L.C., and K.B.; Writing-original draft preparation, L.C., and K.B.; Writing—reviewing and editing, L.C., K.B., and L.O. All authors have read and agreed to the published version of the manuscript.

Funding: This research has been funded by the Pre-application research into innovative medicines and medical technologies project (Reg. No. CZ.02.1.01/0.0/0.0/18_069/0010046) co-funded by the European Union.

Acknowledgments: The authors wish to thank Gerald Blunden for critical reading of the manuscript and corrections of English.

Conflicts of Interest: The authors declare no conflict of interest.

\section{References}

1. Nair, J.J.; van Staden, J. Pharmacological and toxicological insights to the South African Amaryllidaceae. Food Chem. Toxicol. 2013, 62, 262-275. [CrossRef]

2. Jin, Z. Amaryllidaceae and Sceletium alkaloids. Nat. Prod. Rep. 2016, 33, 1318-1343. [CrossRef] [PubMed]

3. Fennell, C.; van Staden, J. Crinum species in traditional and modern medicine. J. Ethnopharmacol. 2001, 78, 15-26. [CrossRef]

4. Havelek, R.; Muthna, D.; Tomsik, P.; Kralovec, K.; Seifrtova, M.; Cahlikova, L.; Hostalkova, A.; Safratova, M.; Perwein, M.; Cermakova, E.; et al. Anticancer potential of Amaryllidaceae alkaloids evaluated by screening with a panel of human cells, real-time cellular analysis and Ehrlich tumor-bearing mice. Chem. Biol. Interact. 2017, 275, 121-132. [CrossRef] [PubMed]

5. Vaněčková, N.; Hošt'álková, A.; Šafratová, M.; Kuneš, J.; Hulcová, D.; Hrabinová, M.; Doskočil, I.; Štěpánková, Š.; Opletal, L.; Nováková, L.; et al. Isolation of Amaryllidaceae alkaloids from Nerine bowdenii W. Watson and their biological activities. RSC Adv. 2016, 6, 80114-80120. [CrossRef]

6. Kornienko, A.; Evidente, A. Chemistry, biology, and medicinal potential of narciclasine and its congeners. Chem. Rev. 2008, 108, 1982-2014. [CrossRef] [PubMed]

7. Marco, L.; do Carmo Carreiras, M. Galanthamine, a natural product for the treatment of Alzheimer's disease. Recent Pat. CNS Drug Discov. 2006, 1, 105-111. [CrossRef] [PubMed]

8. Tae, K.; Ko, S. New taxa of the genus Lycoris. Korean J. Plant Taxon. 1993, 23, 233-241. [CrossRef]

9. Tae, K.; Ko, S. A taxonomic study on the genus Lycoris (Amaryllidaceae). Korean J. Plant Taxon. 1996, 26, 19-35. [CrossRef]

10. Ping-Sheng, H.S.U.; Kurita, S.; Zhi-Zhou, Y.U.; Jin-Zhen, L.I.N. Synopsis of the genus Lycoris (Amaryllidaceae). Sida 1994, 16, 301-331.

11. The Plant List. Available online: http://www.theplantlist.org/tpl1.1/search?q=Lycoris (accessed on 10 September 2020).

12. Chen, G.-L.; Tian, Y.-Q.; Wu, J.-L.; Li, N.; Guo, M.-Q. Antiproliferative activities of Amaryllidaceae alkaloids from Lycoris radiata targeting DNA topoisomerase I. Sci. Rep. 2016, 6, 38284. [CrossRef] [PubMed]

13. Feng, T.; Wang, Y.-Y.; Su, J.; Li, Y.; Cai, X.-H.; Luo, X.-D. Amaryllidaceae alkaloids from Lycoris Radiata. Helv. Chim. Acta 2011, 94, 178-183. [CrossRef]

14. Huang, S.-D.; Zhang, Y.; He, H.-P.; Li, S.-F.; Tang, G.-H.; Chen, D.-Z.; Cao, M.-M.; Di, Y.-T.; Hao, X.-J. A new Amaryllidaceae alkaloid from the bulbs of Lycoris radiata. Chin. J. Nat. Med. 2013, 11, 406-410. [CrossRef]

15. Wang, L.; Zhang, X.Q.; Yin, Z.Q.; Wang, Y.; Ye, W.C. Two new Amaryllidaceae alkaloids from the bulbs of Lycoris Radiata. Chem. Pharm. Bull. 2009, 57, 610-611. [CrossRef]

16. Shen, C.-Y.; Xu, X.-L.; Yang, L.-J.; Jiang, J.-G. Identification of narciclasine from Lycoris radiata (L'Her.) Herb. and its inhibitory effect on LPS-induced inflammatory responses in macrophages. Food Chem. Toxicol. 2019, 125, 605-613. [CrossRef]

17. Cao, Z.; Yang, P.; Zhou, Q. Multiple biological functions and pharmacological effects of lycorine. Sci. China Chem. 2013, 56, 1382-1391. [CrossRef] [PubMed]

18. Jin, Z. Amaryllidaceae and Sceletium alkaloids. Nat. Prod. Rep. 2011, 28, 1126-1142. [CrossRef] [PubMed] 
19. Duke, J.A.; Ayensu, E.S. Medicinal Plants of China, 2nd ed.; Reference Publications: Algonac, MI, USA, 1985; p. 398.

20. Chase, M.; Reveal, J.; Fay, M. A subfamilial classification for the expanded asparagalean families Amaryllidaceae, Asparagaceae and Xanthorrhoeaceae. Bot. J. Linn. Soc. 2009, 161, 132-136. [CrossRef]

21. Kilgore, M.B.; Kutchan, T.M. The Amaryllidaceae alkaloids: Biosynthesis and methods for enzyme discovery. Phytochem. Rev. 2016, 15, 317-337. [CrossRef]

22. Armengol, J.B.I.; Berkov, S.; Claveria, L.T.; Pigni, N.B.; de Andrade, J.P.; Martínez, V.; Mahrer, C.C.; Meya, F.V. Chemical and biological aspects of Amaryllidaceae alkaloids. In Recent Advances in Pharmaceutical Sciences; Muñoz-Torrero, D., Ed.; Transworld Research Network: Kerala, India, 2011; pp. 65-100.

23. Desgagne-Penix, I. Biosynthesis of the Amaryllidaceae alkaloids. Plant Sci. Today 2014, 1, 114-120.

24. Berkov, S.; Martínez-Francés, V.; Bastida, J.; Codina, C.; Ríos, S. Evolution of alkaloid biosynthesis in the genus Narcissus. Phytochemistry 2014, 99, 95-106. [CrossRef] [PubMed]

25. Cahlíková, L.; Vaněčková, N.; Safratova, M.; Breiterová, K.; Blunden, G.; Hulcova, D.; Opletal, L. The genus Nerine Herb. (Amaryllidaceae): Ethnobotany, phytochemistry, and biological activity. Molecules 2019, 24, 4238. [CrossRef]

26. Desgagné-Penix, I. Biosynthesis of alkaloids in Amaryllidaceae plants: A review. Phytochem. Rev. 2020, 5, 239-270. [CrossRef]

27. Ang, S.; Liu, X.M.; Huang, X.J.; Zhang, D.M.; Zhang, W.; Wang, L.; Ye, W.C. Four new Amaryllidaceae alkaloids from Lycoris radiata and their cytotoxicity. Planta Med. 2015, 81, 1712-1718. [CrossRef] [PubMed]

28. Yan, H.; Xie, N.; Zhong, C.; Su, A.; Hui, X.; Zhang, X.; Jin, Z.; Li, Z.; Feng, J.; He, J. Aphicidal activities of Amaryllidaceae alkaloids from bulbs of Lycoris radiata against Aphis citricola. Ind. Crop. Prod. 2018, 123, 372-378. [CrossRef]

29. Berkov, S.; Osorio, E.; Viladomat, F.; Bastida, J. Chapter Two-Chemodiversity, chemotaxonomy and chemoecology of Amaryllidaceae alkaloids. In The Alkaloids: Chemistry and Biology; Knölker, H.-J., Ed.; Academic Press: Cambridge, MA, USA, 2020; Volume 83, pp. 113-185.

30. Zhu, Y.-Y.; Li, X.; Yu, H.-Y.; Xiong, Y.-F.; Zhang, P.; Pi, H.-F.; Ruan, H.-L. Alkaloids from the bulbs of Lycoris longituba and their neuroprotective and acetylcholinesterase inhibitory activities. Arch. Pharm. Res. 2014, 38, 604-613. [CrossRef]

31. Jin, A.; Li, X.; Zhu, Y.Y.; Yu, H.Y.; Pi, H.F.; Zhang, P.; Ruan, H.L. Four new compounds from the bulbs of Lycoris aurea with neuroprotective effects against $\mathrm{CoCl}_{2}$ and $\mathrm{H}_{2} \mathrm{O}_{2}$-induced SH-SY5Y cell injuries. Arch. Pharm. Res. 2014, 37, 315-323. [CrossRef]

32. Tian, Y.; Zhang, C.; Guo, M. Comparative analysis of Amaryllidaceae alkaloids from three Lycoris species. Molecules 2015, 20, 21854-21869. [CrossRef]

33. Zhang, X.; Huang, H.; Liang, X.; Huang, H.; Dai, W.; Shen, Y.; Yan, S.; Zhang, W. Analysis of Amaryllidaceae alkaloids from Crinum by high-performance liquid chromatography coupled with electrospray ionization tandem mass spectrometry. Rapid. Commun. Mass Spectrom. 2009, 23, 2903-2916. [CrossRef]

34. Takayama, H.; Kinoshita, E.; Kitajima, M.; Kogure, N. Two new alkaloids from bulbs of Lycoris squamigera. Heterocycles 2009, 77, 1389-1396. [CrossRef]

35. Li, H.; Ma, G.; Xu, Y.; Hong, S. Alkaloids of Lycoris Guangxiensis. Planta Med. 1987, 53, 259-261. [CrossRef] [PubMed]

36. Kihara, M.; Xu, L.; Konishi, K.; Kida, K.; Nagao, Y.; Kobayashi, S.; Shingu, T. Isolation and structure elucidation of a novel alkaloid, incartine, a supposed biosynthetic intermediate, from flowers of Lycoris incarnata. Chem. Pharm. Bull. 1994, 42, 289-292. [CrossRef]

37. Li, X.; Yu, H.Y.; Wang, Z.Y.; Pi, H.F.; Zhang, P.; Ruan, H.L. Neuroprotective compounds from the bulbs of Lycoris radiata. Fitoterapia 2013, 88, 82-90. [CrossRef] [PubMed]

38. Kihara, M.; Konishi, K.; Xu, L.; Kobayashi, S. Alkaloidal constituents of the flowers of Lycoris radiata HERB: Amaryllidaceae. Chem. Pharm. Bull. 1991, 39, 1849-1853. [CrossRef]

39. Jitsuno, M.; Yokosuka, A.; Hashimoto, K.; Amano, O.; Sakagami, H.; Mimaki, Y. Chemical constituents of Lycoris albiflora and their cytotoxic activities. Nat. Prod. Commun. 2011, 6, 1934578X1100600208. [CrossRef]

40. Boit, H.G.; Döpke, W.; Stender, W. Alkaloide aus Hippeastrum rutilum, Lycoris albiflora, Zephyranthes andersoniana und Sternbergia fischeriana. Naturwissenschaften 1958, 45, 390. [CrossRef] 
41. Guo, Y.; Pigni, N.; Zheng, Y.; de Andrade, J.; Torras-Claveria, L.; Borges, W.; Francesc, V.; Codina, C.; Bastida, J. Analysis of bioactive Amaryllidaceae alkaloid profiles in Lycoris species by GC-MS. Nat. Prod. Commun. 2014, 9, 1081-1086. [CrossRef]

42. Boit, H.-G.; Ehmke, H. XVI. Mitteil. über Amaryllidaceen-Alkaloide. Alkaloide von Nerine corusca, N. flexuosa, Pancratium illyricum, Lycoris aurea und L. Incarnata. Chem. Ber. 1957, 90, 369-373. [CrossRef]

43. Mu, H.M.; Wang, R.; Li, X.D.; Jiang, Y.M.; Peng, F.; Xia, B. Alkaloid accumulation in different parts and ages of Lycoris Chinensis. Z. Nat. C 2010, 65, 458-462. [CrossRef] [PubMed]

44. Wu, W.-M.; Zhu, Y.-Y.; Li, H.-R.; Yu, H.-Y.; Zhang, P.; Pi, H.-F.; Ruan, H.-L. Two new alkaloids from the bulbs of Lycoris sprengeri. J. Asian Nat. Prod. Res. 2014, 16, 192-199. [CrossRef] [PubMed]

45. Toriizuka, Y.; Kinoshita, E.; Kogure, N.; Kitajima, M.; Ishiyama, A.; Otoguro, K.; Yamada, H.; Ōmura, S.; Takayama, H. New lycorine-type alkaloid from Lycoris traubii and evaluation of antitrypanosomal and antimalarial activities of lycorine derivatives. Bioorganic Med. Chem. 2008, 16, 10182-10189. [CrossRef] [PubMed]

46. Wang, L.; Yin, Z.-Q.; Cai, Y.; Zhang, X.-Q.; Yao, X.-S.; Ye, W.-C. Amaryllidaceae alkaloids from the bulbs of Lycoris Radiata. BioChem. Syst. Ecol. 2010, 38, 444-446. [CrossRef]

47. Hao, B.; Shen, S.F.; Zhao, Q.J. Cytotoxic and antimalarial Amaryllidaceae alkaloids from the bulbs of Lycoris radiata. Molecules 2013, 18, 2458-2468. [CrossRef]

48. Liu, Z.-M.; Huang, X.-Y.; Cui, M.-R.; Zhang, X.-D.; Chen, Z.; Yang, B.-S.; Zhao, X.-K. Amaryllidaceae alkaloids from the bulbs of Lycoris radiata with cytotoxic and anti-inflammatory activities. Fitoterapia 2015, 101, 188-193. [CrossRef] [PubMed]

49. Liao, N.; Ao, M.; Zhang, P.; Yu, L. Extracts of Lycoris aurea induce apoptosis in murine sarcoma S180 cells. Molecules 2012, 17, 3723-3735. [CrossRef]

50. Song, J.-H.; Zhang, L.; Song, Y. Alkaloids from Lycoris aurea and their cytotoxicities against the head and neck squamous cell carcinoma. Fitoterapia 2014, 95, 121-126. [CrossRef]

51. Deng, B.; Ye, L.; Yin, H.; Liu, Y.; Hu, S.; Li, B. Determination of pseudolycorine in the bulb of Lycoris radiata by capillary electrophoresis combined with online electrochemiluminescence using ultrasonic-assisted extraction. J. Chromatogr. B 2011, 879, 927-932. [CrossRef]

52. Cao, P.; Pan, D.S.; Han, S.; Yu, C.Y.; Zhao, Q.J.; Song, Y.; Liang, Y. Alkaloids from Lycoris caldwellii and their particular cytotoxicities against the astrocytoma and glioma cell lines. Arch. Pharm. Res. 2013, 36, 927-932. [CrossRef]

53. Yun, Y.S.; Tajima, M.; Takahashi, S.; Takahashi, Y.; Umemura, M.; Nakano, H.; Park, H.S.; Inoue, H. Two alkaloids from bulbs of Lycoris sanguinea MAXIM. suppress PEPCK expression by inhibiting the phosphorylation of CREB. Phytother. Res. 2016, 30, 1689-1695. [CrossRef]

54. Pi, H.F.; Zhang, P.; Ruan, H.L.; Zhang, Y.H.; Sun, H.D.; Wu, J.Z. A new alkaloid from Lycoris aurea. Chin. Chem. Lett. 2009, 20, 1319-1320. [CrossRef]

55. Nair, J.J.; van Staden, J. Cytotoxicity studies of lycorine alkaloids of the Amaryllidaceae. Nat. Prod. Commun. 2014, 9, 1934578X1400900834. [CrossRef]

56. Habartová, K.; Cahlíková, L.; Řezáčová, M.; Havelek, R. The biological activity of alkaloids from the Amaryllidaceae: From cholinesterases inhibition to anticancer activity. Nat. Prod. Commun. 2016, 11, 1934578X1601101038. [CrossRef]

57. Nair, J.J.; Rárová, L.; Strnad, M.; Bastida, J.; van Staden, J. Mechanistic insights to the cytotoxicity of Amaryllidaceae alkaloids. Nat. Prod. Commun. 2015, 10, 1934578X1501000138. [CrossRef]

58. Nair, J.J.; Bastida, J.; Viladomat, F.; van Staden, J. Cytotoxic agents of the crinane series of Amaryllidaceae alkaloids. Nat. Prod. Commun. 2013, 8, 1934578X1300800501. [CrossRef]

59. McNulty, J.; Nair, J.J.; Bastida, J.; Pandey, S.; Griffin, C. Structure-activity studies on the lycorine pharmacophore: A potent inducer of apoptosis in human leukemia cells. Phytochemistry 2009, 70, 913-919. [CrossRef] [PubMed]

60. Evdokimov, N.M.; Lamoral-Theys, D.; Mathieu, V.; Andolfi, A.; Frolova, L.V.; Pelly, S.C.; van Otterlo, W.A.; Magedov, I.V.; Kiss, R.; Evidente, A.; et al. In search of a cytostatic agent derived from the alkaloid lycorine: Synthesis and growth inhibitory properties of lycorine derivatives. Bioorganic Med. Chem. 2011, 19, 7252-7261. [CrossRef] 
61. Griffin, C.; Sharda, N.; Sood, D.; Nair, J.; McNulty, J.; Pandey, S. Selective cytotoxicity of pancratistatin-related natural Amaryllidaceae alkaloids: Evaluation of the activity of two new compounds. Cancer Cell Int. 2007, 7, 10. [CrossRef] [PubMed]

62. Cahlíková, L.; Kawano, I.; Rezáčová, M.; Blunden, G.; Hulcova, D.; Havelek, R. The Amaryllidaceae alkaloids haemanthamine, haemanthidine and their semisynthetic derivatives as potential drugs. Phytochem. Rev. 2020, 26, 1519-1524. [CrossRef]

63. Lefranc, F.; Sauvage, S.; Goietsenoven, G.; Mégalizzi, V.; Lamoral-Theys, D.; Debeir, O.; Spiegl-Kreinecker, S.; Berger, W.; Mathieu, V.; Decaestecker, C.; et al. Narciclasine, a plant growth modulator, activates Rho and stress fibers in glioblastoma cells. Mol. Cancer 2009, 8, 1739-1750. [CrossRef]

64. Pettit, G.R.; Pettit, G.R.; Backhaus, R.A.; Boyd, M.R.; Meerow, A.W. Antineoplastic agents, 256. Cell growth inhibitory isocarbostyrils from Hymenocallis. J. Nat. Prod. 1993, 56, 1682-1687. [CrossRef]

65. Van Goietsenoven, G.; Hutton, J.; Becker, J.-P.; Lallemand, B.; Robert, F.; Lefranc, F.; Pirker, C.; Vandenbussche, G.; Van Antwerpen, P.; Evidente, A.; et al. Targeting of eEF1A with Amaryllidaceae isocarbostyrils as a strategy to combat melanomas. FASEB J. 2010, 24, 4575-4584. [CrossRef] [PubMed]

66. Gopalakrishnan, R.; Matta, H.; Choi, S.; Chaudhary, P. Narciclasine, an isocarbostyril alkaloid, has preferential activity against primary effusion lymphoma. Sci. Rep. 2020, 10, 5712. [CrossRef] [PubMed]

67. Dumont, P.; Ingrassia, L.; Rouzeau, S.; Ribaucour, F.; Thomas, S.; Roland, I.; Darro, F.; Lefranc, F.; Kiss, R. The Amaryllidaceae isocarbostyril narciclasine induces apoptosis by activation of the death receptor and/or mitochondrial pathways in cancer cells but not in normal fibroblasts 1. Neoplasia 2007, 9, 766-776. [CrossRef] [PubMed]

68. Van Goietsenoven, G.; Mathieu, V.; Lefranc, F.; Kornienko, A.; Evidente, A.; Kiss, R. Narciclasine as well as other Amaryllidaceae isocarbostyrils are promising GTP-ase targeting agents against brain cancers. Med. Res. Rev. 2013, 33, 439-455. [CrossRef]

69. Stark, A.; Schwenk, R.; Wack, G.; Zuchtriegel, G.; Hatemler, M.; Bräutigam, J.; Schmidtko, A.; Reichel, C.; Bischoff, I.; Fürst, R. Narciclasine exerts anti-inflammatory actions by blocking leukocyte-endothelial cell interactions and down-regulation of the endothelial TNF receptor 1. FASEB J. 2019, 33, 8771-8781. [CrossRef]

70. Nordberg, A.; Ballard, C.; Bullock, R.; Darreh-Shori, T.; Somogyi, M. A review of butyrylcholinesterase as a therapeutic target in the treatment of Alzheimer's disease. Prim. Care Companion CNS Disord. 2013, 15. [CrossRef]

71. Yu, W.; Hao, W.; Hong-zhuan, C. AChE Inhibition-based multi-target-directed ligands, a novel pharmacological approach for the symptomatic and disease-modifying therapy of Alzheimer's Disease. Curr. Neuropharmacol. 2016, 14, 364-375.

72. De Vita, D.; Pandolfi, F.; Ornano, L.; Feroci, M.; Chiarotto, I.; Sileno, I.; Pepi, F.; Costi, R.; Di Santo, R.; Scipione, L. New N,N-dimethylcarbamate inhibitors of acetylcholinesterase: Design, synthesis and biological evaluation. J. Enzym. Inhib. Med. Chem. 2016, 31, 106-113. [CrossRef]

73. Al Mamun, A.; Maříková, J.; Hulcová, D.; Janoušek, J.; Šafratová, M.; Nováková, L.; Kučera, T.; Hrabinová, M.; Kuneš, J.; Korábečný, J.; et al. Amaryllidaceae alkaloids of belladine type from Narcissus pseudonarcissus cv. Carlton as new selective inhibitors of butyrylcholinesterase. Biomolecules 2020, 10, 800. [CrossRef]

74. Hulcová, D.; Maříková, J.; Korábečný, J.; Hošt'álková, A.; Jun, D.; Kuneš, J.; Chlebek, J.; Opletal, L.; De Simone, A.; Nováková, L.; et al. Amaryllidaceae alkaloids from Narcissus pseudonarcissus L. cv. Dutch Master as potential drugs in treatment of Alzheimer's disease. Phytochemistry 2019, 165, 112055. [CrossRef]

75. Moreno, R.; Tallini, L.R.; Salazar, C.; Osorio, E.H.; Montero, E.; Bastida, J.; Oleas, N.H.; Acosta León, K. Chemical profiling and cholinesterase inhibitory activity of five Phaedranassa Herb. (Amaryllidaceae) species from Ecuador. Molecules 2020, 25, 2092. [CrossRef] [PubMed]

76. T Tallini, L.R.; Bastida, J.; Cortes, N.; Osorio, E.H.; Theoduloz, C.; Schmeda-Hirschmann, G. Cholinesterase inhibition activity, alkaloid profiling and molecular docking of Chilean Rhodophiala (Amaryllidaceae). Molecules 2018, 23, 1532. [CrossRef] [PubMed]

77. Tiwari, M.K.; Chaudhary, S. Artemisinin-derived antimalarial endoperoxides from bench-side to bed-side: Chronological advancements and future challenges. Med. Res. Rev. 2020, 40, 1220-1275. [CrossRef] 
78. Dedryver, C.-A.; Le Ralec, A.; Fabre, F. The conflicting relationships between aphids and men: A review of aphid damage and control strategies. Comptes Rendus Biol. 2010, 333, 539-553. [CrossRef] [PubMed]

79. Liu, B.; Gao, X.; Zheng, B. Effects of sublethal doses of anticholinesterase agents on toxicity of insecticides and their induction to acetylcholinesterase (AChE) activity in Helicoverpa armigera. Acta Entomol. Sin. 2003, $46,691-696$.

Publisher's Note: MDPI stays neutral with regard to jurisdictional claims in published maps and institutional affiliations.

(C) 2020 by the authors. Licensee MDPI, Basel, Switzerland. This article is an open access article distributed under the terms and conditions of the Creative Commons Attribution (CC BY) license (http://creativecommons.org/licenses/by/4.0/). 